Research Article

\title{
Control Policy Mix in Measles Transmission Dynamics Using Vaccination, Therapy, and Treatment
}

\author{
Jaharuddin (iD) and Toni Bakhtiar (i) \\ Department of Mathematics, Faculty of Mathematics and Natural Sciences, IPB University, Bogor 16680, Indonesia \\ Correspondence should be addressed to Jaharuddin; jaharmath@gmail.com
}

Received 7 October 2019; Revised 20 January 2020; Accepted 7 February 2020; Published 12 March 2020

Academic Editor: Hans Engler

Copyright (C) 2020 Jaharuddin and Toni Bakhtiar. This is an open access article distributed under the Creative Commons Attribution License, which permits unrestricted use, distribution, and reproduction in any medium, provided the original work is properly cited.

\begin{abstract}
This paper considers a deterministic model for the dynamics of measles transmission in a population divided into six classes with respect to the disease states: susceptible, vaccinated, exposed, infected, treated, and recovered. First, we investigate the dynamical properties of the SVEITR model such as its equilibrium points, their stability, and parameter sensitivity by applying constant controls. Criteria for determining the stability of disease-free and endemic equilibrium points are provided in terms of basic reproduction number. The model is then extended by incorporating vaccination, therapy, and treatment rates as time-dependent control variables representing the level of coverages. Application of Pontryagin's maximum principle provides the necessary conditions that must be satisfied for the existence of optimal controls aiming at minimization of the number of exposed and infected individuals simultaneously with the control effort. Numerical simulations that were carried out using the backward sweep method and Runge-Kutta scheme suggest that optimal controls under moderate and high scenarios can effectively reduce the cases of measles. In particular, the moderate scenario that utilizes the existing coverage level of $86 \%$ for MCV1 and $69 \%$ for MCV2 can degrade the cost functional by $47 \%$ of the low scenario. Meanwhile, high scenario that takes the 2020 target of $96 \%$ as coverage only makes a slight difference in reducing the number of exposed and infected individuals.
\end{abstract}

\section{Introduction}

Measles as a highly contagious and serious viral disease has been known for centuries. The formal story of measles has begun when the first written accounts of this disease were published by a Persian physician in the ninth century. But until now, measles is still a scourge in many countries. Although it was declared eradicated in the US in 2000, i.e., there is an absence of continuous disease spread for more than 12 months, and it appears to have reemerged due to a decreased level of inoculation. It was reported that measles cases that occurred in 2019 were the highest in the last 25 years. Major outbreaks were also notified by other countries in the globe such as Madagascar, Congo, Angola, Sudan, South Sudan, Cameroon, Chad, and Nigeria in Africa, Ukraine in Eastern Europe, Kazakhstan in Central Asia, and Philippines and Thailand in Southeast Asia, making 2019 a record-setting year [1]. A report by the World Health Organization (WHO) states that during the first semester of
2019, a total of 182 countries reported 364,811 cases of measles: about the same number of countries but with almost threefold increase in the number of cases compared to the same period in the previous year. Significant increase in measles cases occurred in African, the European, and the Western Pacific regions [2].

Measles is caused by paramyxovirus, an RNA virus classified under the Morbillivirus genus of the Paramyxoviridae family. Initially beginning with flu-like symptoms which appear 7 to 14 days after contact with the virus, measles presents with Koplik spots inside the mouth and rashes which appear 2 to 5 days after the first symptoms, starting on the head and face and spreading through the body. Measles can be dangerous, especially for babies, young children under five, and adults older than 20 years of age. Measles is also infectious for a few days after rash onset, commonly accompanyed by high fever, red and watery eyes, cough, and runny nose. About one-third of reported measles cases have one or more complications, 
ranging from common complications such as ear infections (otitis media) and diarrhea to serious complications such as pneumonia and acute encephalitis [3, 4]. Measles virus is transmitted primarily person to person via large respiratory droplets by coughing and sneezing, close personal contact or via direct contact with infected nasal or throat secretions. Before a measles vaccine was available, infection caused by measles virus was nearly universal during childhood, and more than ninety percent of persons were self-immune by the age of fifteen. Due to the availability of a safe, effective, and inexpensive vaccine as well as accelerated immunization activities, measles-induced fatalities have declined by $80 \%$ globally in latest years, from 545,000 deaths in 2000 to 111,000 deaths in 2017 [2]. However, in many developing countries, particularly in parts of Africa and Asia, measles is still commonly found and is a leading cause of death.

Tremendous efforts have globally been conducted by various institutions and regionally by many countries in combating measles. The Measles \& Rubella Initiative (M\&R Initiative) was launched in 2001 as a global partnership led by the American Red Cross, United Nations Foundation, Centers for Disease Control and Prevention (CDC), UNI$\mathrm{CEF}$, and WHO to ascertain that no child dies from measles or is born with congenital rubella syndrome (CRS). The objective is achieved by providing people in all communities more equitable access to existing vaccines such that coverage of vaccination even further to $95 \%$ by 2020 . In 2012, the World Health Assembly (WHA) endorsed the Global Vaccine Action Plan (GVAP), aiming at eliminating measles in four WHO regions by 2015 and in five regions by 2020 . The Global Measles and Rubella Strategic Plan 2012-2020 outlines the strategy that needs to be fully implemented by countries, the M\&R Initiative and its partners, in achieving a world without measles, rubella, and CRS endorsed by the WHA [5]. For the future action plan, an initial draft of Immunization Agenda 2030 has already been disseminated broadly for public consultation.

Africa has made vast advancement in its effort to protect children from diseases and to control the disease, intensifying its regional measles vaccination coverage from $56 \%$ in $2001,73 \%$ in 2008 , and $85 \%$ in 2010 , even though in recent years, however, the Africa region has experienced stagnation in vaccination coverage [6]. In particular, the Republic of Kenya is one of countries in the continent that implements remarkable approaches in their fight against measles. Kenya, as a supporter of the WHA and Measles Elimination 2020 resolutions, has adopted several innovations to eliminate measles such as Supplementary Immunization Activities (SIAs). This effort includes the application of auto-disable (AD) syringes, implementation of injection safety support (INS) to scale up safe injection, introduction of measles-rubella (MR) combined vaccine, case-based surveillance using real-time polymerase chain reaction (PCR), serological tests for immunoglobulin class M (IgM) detection, and epidemiological link as well as the use of SMS reminder system for routine immunization [7]. After ten years without outbreaks, a measles epidemic was reported by Madagascar on
October 2018 with more than 117,000 cases causing more than 1,000 deaths. Madagascar, with support from the WHO, GAVI The Vaccine Alliance, M\&R Initiative, UNICEF, and the USAID, then conducted emergency vaccination campaigns ultimately targeting all 7 million children in the country aged 6 months to 9 years. Now, the number of measles and fatalities in Madagascar relatively declines [2].

Considerable efforts have also been undertaken by countries in other regions. Member countries of the WHO South-East Asia region has adopted measles elimination and rubella control as one of the eight regional flagship priority programs since 2014 and has resolved to eliminate measles and rubella by 2023 . All countries agreed to strengthening the immunization systems, establishing a highly sensitive laboratory supported case-based surveillance system, and deploying political, societal, and financial support to control the virus transmission. During the 2014-2017 period, there has been a $23 \%$ decline in mortality due to measles in the region [8]. In the European region, the coverage of immunization for the second dose of measles vaccine in 2018 was $91 \%$, meaning that the level of coverage has reached a record high in the region. However, based on a risk assessment and dramatic increase on measles cases, i.e., 160,000 cases and over 100 measles-related deaths during 2018-2019 period, the WHO activated a Grade 2 emergency response to measles spreading in the region. It means that WHO/Europe will continue to work with all 53 member states in the region to improve immunization and disease surveillance technologies and to respond to measles outbreaks [9]. However, in the Americas region, the total number of confirmed cases of measles in 2019 increase by 70\% compared to 2018 with United States and Brazil providing the most cases. Given the situation, the Pan American Health Organization (PAHO) and WHO reinforce the recommendations to all member states to focus on achieving the shared goal of measles elimination. This effort includes maintaining immunization coverage of $95 \%$ as well as maintaining vaccine stock, identifying migratory flows, implementing immunization for migrant populations, and strengthening epidemiological surveillance including that in border areas [10].

A substantial number of initiatives have been carried out by policy and health decision-makers to eradicate measles. For continuous improvement in the future, quantitative methods are required to examine the impact and cost-effectiveness of these efforts [11]. As a vigorous language that compels us to be logically consistent and explicit about assumptions, in recent years, mathematical models have been increasingly utilized to assist policy makers in predicting the effect of various factors and intervention. Mathematical modeling is an invaluable tool for decision-making, particularly when direct assessment of interventions is not an option. Modeling can also be used to estimate future outcomes that are otherwise difficult to evaluate [12]. Mathematical modeling is formulated by a set of rules, mostly by means of differential or difference equations, to describe the real-life dynamic of the process. The model is developed based on key 
assumptions about the relationship between input and output. The more realistic the assumptions applied, the more the variables and parameters involved so that the model considered is also more complex [13]. In the epidemiology of measles, the mathematical model has become a viable approach to understand the biological process of the disease [14, 15]. It can also be harnessed to assess the effect of public health intervention strategies [16-19] and recommend the best course of measure in combating measles including suggesting the optimal schedule of vaccination $([20-22])$.

This present paper discusses the modeling and control of measles transmission dynamics. We develop a compartmental model of measles based on a set of quite general assumptions. The model includes the existence of exposed and treated subpopulations as well as a class of vaccinated individuals accommodating two-dose routine policy. This setting, however, enables us to comprehensively assess the nature of the disease from the dynamical behavior and control perspectives. The first part of this paper concerns with the dynamical properties of the measles model such as equilibrium points, basic reproduction number, and analysis of stability. However, the second part deals with the optimal control model of measles dynamic, a framework that allows us to study the effect of intervention strategies in controlling the spread of measles. Particularly, we evaluate a series of scenarios regarding the coverage of vaccination, the rate of measles therapy, and the rate of treatment.

This paper is organized into six sections. After introductory part and research background in Section 1, we present in Section 2 a brief literature review of related works in the topic. In Sections 3 and 4, we introduce the development of the model and assumptions. The first part deals with the so-called dynamical model with constant control. We analyze the dynamic behavior and stability of disease-free and endemic equilibrium points and provide two stability theorems. However, the second part discusses the measles transmission model with optimal control. Section 5 is devoted to the results and discussion. Concluding remarks and direction for future research are provided in Section 6.

\section{Related Works}

The number of research studies that employ mathematical modeling to study the dynamics of infectious disease has rapidly increased over the last two decades. The research foci in this area are ranging from the study of respiratory diseases such as measles, influenza, and tuberculosis; vector-borne diseases such as malaria, Ebola, zikav, and dengue; to sexually transmitted diseases such as HIV/AIDS (see for instance the works of Beay [23], Reynolds et al. [24], Mitchell and Ross [25], Egonmwan and Okuonghae [26], Nkamba et al. [27], Bakary et al. [28], Irwan et al. [29], Akgül et al. [30], Ainisa et al. [31], Carvalho et al. [32], Omondi et al. [33], and Chong et al. [34]). Mathematical modeling is usually used to characterize the epidemiological parameters of disease during outbreaks and to evaluate the effectiveness and schedule of various prevention and control strategies, considering limited resource availability [35]. In general, the primary objective of mathematical study in epidemiology is to reinforce understanding of the interplay between variables that govern the course of infection within an individual and those that control the behavior of infection within population as a whole.

There are abundant references on modeling of measles. Existing studies generally relate to modeling the dynamic behavior of measles and its stability characteristics, sensitivity analysis to model parameters, and evaluation and prediction of the effectiveness of control strategies in the form of optimal control models. Roberts and Tobias [36] conducted modeling to predict and prevent the spread of measles in New Zealand. In their study, a deterministic SIR model was utilized to describe the spread of measles. This model successfully predicted an epidemic in 1997 and, at that time, the decision to carry out the MMR immunization campaign was very important in New Zealand. Ochoche and Gweryina [37] used a mathematical model of measles transmission that involves two phases of infection. They divided the population into suspected, infected, and recovered compartments. Their study revealed that the disease would definitely be eliminated if all suspected populations were vaccinated. But this is not efficient since measles is mostly found in children aged five years or younger. Then, it was suggested that the measles vaccine should be given in such a way that no child can enter school without evidence of at least a two doses of measles vaccination. Momoh et al. [16] developed a mathematical model describing the effect of control effort to the spread of measles. An SEIR (suspected, exposed, infected, and recovered) model was exploited to determine individuals exposed to latent periods based on numerical simulations. This model was later developed by Momoh et al. [17] to study the effect of vaccination on the dynamics of the spread of measles. In their study, the total population was categorized into five classes, namely, suspected, exposed, infected, recovered, and immune infants. They estimated the optimal vaccine coverage needed to control the spread of measles. MacIntyre et al. [38] introduced a mathematical model involving Measles Control Campaign (MCC) regarding the potential of measles transmission in Australia. In this study, the population was divided into five age groups. They used the survey results and estimated the vaccine coverage to calculate basic reproduction number after the measles control campaign. The results showed that MCC had a significant influence on the dynamics of the spread of measles in Australia. In addition, ongoing efforts are needed to improve the MMR (measles, mumps, rubella) vaccine so that measles transmission can be eliminated. Bakare et al. [14] also propose the use of SEIR model to portray the dynamics of the spread of measles without discussing the effects of the vaccination control variables. Bolarian [15] modified the SEIR model by adding vaccinated compartments. The research was aiming at determination of vaccination coverage and dosage for removing measles in a population. Mossong and Muller [39] used a mathematical model featuring individuals who experienced decreased immunity in the vaccinated population were suspected. In this model, waning immunity can bring 
back measles. This event is certainly realistic. Fred et al. [18] made use of an SEIR model with the presence of control variables in the form of vaccination. They recommended the introduction of a mass vaccination program and an increase in early detection of measles cases to minimize the chance of an outbreak.

Immunization is a highly effective healthcare intervention of measles even though in high-burden settings, it remains a challenge due primarily to low quality of healthcare infrastructure and access. Pang et al. [22] developed a SEIR model with vaccination to investigate its effect in controlling the transmission of measles among people. Aldila and Asrianti [40] discussed a modified SVIQR (susceptible, vaccinated, infected, quarantine, and recovered) deterministic model for measles transmission, equipped with two intervention strategy, namely, two times vaccination and quarantine. Two compartments for the vaccinated population were introduced to categorize human population who get vaccinated once and twice. It is confirmed in this study that receiving two doses of measles vaccine can reduce the disease transmission much better rather than only with one dose of vaccination. Another study by Beay [23] analyzed the effects of treatment and quarantine to reduce the spread of measles through a SIQR (susceptible, infected, quarantine, and recovered) model. The study by Wallinga et al. [41] attempted to investigate why measles outbreaks still occur in vaccinated populations. In particular, the study wanted to identify the rates of vaccination that affected the chances of an outbreak occurring and what the effect of people being unvaccinated was. The dynamic of measles infection transmission in the form of age-stratified compartmental model was developed by Verguet et al. [42] aiming at exploration of the frequency of SIAs in order to achieve measles control in selected countries. Beyond the susceptible, infected, and recovered compartments, the model contains three vaccinated classes, namely, vaccinated susceptible, vaccinated infected, and vaccinated recovered. It indicates that the measles transmission in any of the countries with high measles burden cannot be controlled by a single SIA. However, the measles outbreaks can still be prevented by regular SIAs at high coverage levels. The optimal scheduling of SIAs is determined by population demographics and the coverage of existing routine immunization.

Research relating to the interconnection between immunization coverage, vaccination schedules, and the dynamics of measles transmission has been carried out by many researchers. It is reported in Portnoy et al. [43] that the immunization coverage has been increased in the $21 \mathrm{st}$ century and the global burden of measles mortality has been substantially reduced, followed by the fact that the casefatality ratios for measles have seen considerable declines since the 1990s. According to this research, the estimate for the mean of case-fatality ratio is $2.2 \%$ and projected to be a $1.3 \%$ in 2016-2030. It had been recommended by Thakkar et al. [20] that the burden of measles can also be decreased by optimizing campaign timing. In developed countries like England, the measles vaccination is usually carried out in children around age 1 and again before starting school at around age 5 [44]. In low-income countries, current evidence suggests an earlier timing of immunization. The optimal timing for a single dose of measles vaccine would probably have been 6 or 7 months of age and at 12 months of age in countries with limited measles transmission. An early two-dose schedule at 4-5 months and 9 months of age should have also been even better in terms of reducing child mortality [45]. On the other side, a study by McKee et al. [21] concluded that the optimal timing for immunization of the first dose of measles vaccine depends on the timing and coverage of both doses, and vice versa.

In this work, we examine the dynamics and control of measles transmission. We formulate the interaction between subpopulations in community within a framework of the SVEITR model, i.e., an SEIR model equipped with vaccinated and treated compartments. We investigate the course of the model under constant and optimal controls. We use the model to simulate the data of measles cases reported in Indonesia. The data of measles cases used are sourced from Directorate General of Disease Prevention and Control, Ministry of Health, Republic of Indonesia (2018). In particular, we explore the effect of the change in immunization coverage on the control objective of minimization of the number of exposed and infected individuals simultaneously with the control effort.

\section{Model with Constant Control}

Measles can be prevented with vaccine. Despite the existence of a safe, effective, and affordable vaccine since 1963, measles cases continue to occur for a variety of reasons [7]. One of the key strategies in eliminating measles is achieving and maintaining the high levels of community immunity by providing a wide range of vaccination coverage with two doses of measles containing vaccines. It is recommended that all children obtain two doses of measles vaccine, starting with the first dose on or after the first birthday and the second dose at 4-6 years of age or at least 28 days after the first dose [3]. Measles is a severe disease that can lead to complications and fatality. The lack of adequate health care for children with measles can increase the probability that untreated complications will progress to high-risk complications and ultimately to death.

Motivated by the facts that vaccination and treatment are among key strategies in the management and mitigation of measles, we propose an extended compartmental model by adding vaccinated and treated classes into the standard SEIR model. Thus, to describe the dynamics of the spread of measles, we consider a population that consists of six subpopulations, namely, susceptible $(S)$, vaccinated $(V)$, exposed $(E)$, infected $(I)$, treated $(T)$, and recovered $(R)$. The compartmental diagram of our SVEITR model is given in Figure 1, and all variables and parameters of the model are described in Table 1.

3.1. Model Assumptions. The SVEITR compartmental model depicted in Figure 1 is developed under the following assumptions: 


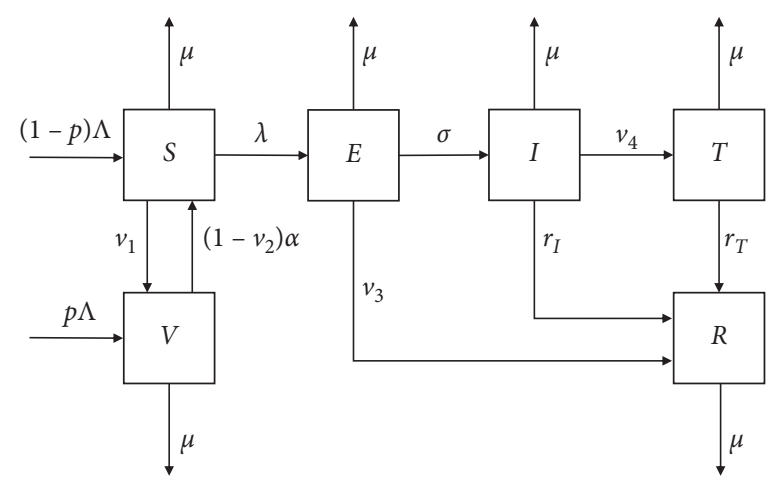

Figure 1: Compartmental model of measles transmission.

TABLE 1: Description parameters of the model.

\begin{tabular}{|c|c|c|}
\hline Variable & Description & Unit \\
\hline$S(t)$ & The number of susceptible individuals at time $t$ & People \\
\hline$V(t)$ & The number of vaccinated individuals at time $t$ & People \\
\hline$E(t)$ & The number of exposed individuals at time $t$ & People \\
\hline$I(t)$ & The number of infected individuals at time $t$ & People \\
\hline$T(t)$ & The number of treated individuals at time $t$ & People \\
\hline$R(t)$ & The number of recovered individuals at time $t$ & People \\
\hline$N(t)$ & Total population at time $t$ & People \\
\hline Parameter & Description & Unit \\
\hline$\Lambda$ & The rate at which new individuals enter the population & People/day \\
\hline$p$ & A fraction of $\wedge$ which is vaccinated or having maternal immunity & - \\
\hline$\mu$ & Natural death rate & $1 /$ day \\
\hline$\alpha$ & The rate of waning of vaccine efficacy after a single dose & 1/day \\
\hline$\lambda$ & The force of infection & day/people \\
\hline$\sigma$ & The rate of exposed individuals become infective & $1 /$ day \\
\hline$v_{1}$ & Proportion of susceptible individuals who receive the first dose of measles containing vaccine & $1 /$ day \\
\hline$v_{2}$ & Proportion of vaccinated individuals who obtain the second dose of vaccine & - \\
\hline$v_{3}$ & The rate of measles therapy & $1 /$ day \\
\hline$v_{4}$ & The treatment rate for infected individuals & $1 /$ day \\
\hline$r_{I}$ & The natural recovery rate & $1 /$ day \\
\hline$r_{T}$ & The recovery rate for treated individuals & $1 /$ day \\
\hline
\end{tabular}

(1) Population is divided into six compartments (susceptible, vaccinated, exposed, infected, treated, and recovered), and it is possible to classify each member of population into a compartment based on his/her state towards disease. The number of populations in compartments at time $t$ is given by $S(t), V(t)$, $E(t), I(t), T(t)$, and $R(t)$, respectively. The total population at time $t$ is denoted by $N(t):=$ $S(t)+V(t)+E(t)+I(t)+T(t)+R(t)$.

(2) We consider an open population with vital dynamics, where new birth including immigrants can provide more susceptible and vaccinated individuals to the population with constant rate $\Lambda \geq 0$. It is assumed that some immigrants and newborns with proportion $p \in[0,1]$ have been vaccinated or have maternally been immunized. The natural mortality rate is also constant $\mu \geq 0$ for all compartments.
(3) Susceptible individuals receive the first dose of measles containing vaccine with proportion $v_{1} \in[0,1]$. It is assumed that the rate of waning of vaccine efficacy after a single dose is $\alpha$ and the proportion of individuals who obtain the second dose of vaccine is $v_{2} \in[0,1]$.

(4) The force of infection $\lambda \geq 0$, which is defined as the instantaneous per capita rate at which susceptible individuals acquire infection, is assumed to be ageindependent and given by

$$
\lambda=\frac{\beta\left(c_{I} I+c_{T} T\right)}{N},
$$

where $\beta \geq 0$ is the effective contact rate, i.e., a composite parameter measuring the contact rate and the probability of transmission upon contact. The term $\left(c_{I} I+c_{T} T\right) / N$ denotes the density or 
prevalence of infected and treated individuals in the population, where $c_{I} \in[0,1]$ and $c_{T} \in[0,1]$ are, respectively, the reduction in transmissibility of infected and treated individuals who fail treatment relative to infectious individuals [46]. Thus, the incidence (the number of new cases per unit time) is given by $\lambda S$.

(5) When latent period ends, exposed individuals may either progress to the infected class at rate $\sigma \geq 0$ or get healing due to measles therapy at rate $v_{3} \geq 0$.

(6) The treatment rate for infected individuals is $v_{4} \geq 0$. Part of the infective population can be recovered without treatment with natural recovery rate $r_{I} \geq 0$. Treated individuals can get recovery at rate $r_{T} \geq 0$.

Implementation of abovementioned assumptions allows us to extend the work of Onyejekwe and Kebede [19], Verguet et al. [42], Pang et al. [22], Simons et al. [47], and Edward et al. [48] by introducing vaccinated or treated classes. To some extent, our model offers an option in applying vaccination policy, compared to that of Aldila and Asrianti [40], which arrange vaccination compartments in series. In this issue, our model shares similarities with models developed by Obumneke et al. [49] and Viriyapong and Ridbamroong [50].

3.2. Model Formulation. From the schematic diagram in Figure 1, we derive the equations of motion of the model, which capture the dynamics of the measles transmission. The SVEITR model can then be represented by the following set of ordinary nonlinear differential equations as follows:

$$
\begin{aligned}
& \frac{\mathrm{d} S}{\mathrm{~d} t}=(1-p) \Lambda+\left(1-v_{2}\right) \alpha V-\left(v_{1}+\mu+\lambda\right) S, \\
& \frac{\mathrm{d} V}{\mathrm{~d} t}=p \Lambda-\left(\mu+\left(1-v_{2}\right) \alpha\right) V+v_{1} S, \\
& \frac{\mathrm{d} E}{\mathrm{~d} t}=\lambda S-\left(v_{3}+\mu+\sigma\right) E, \\
& \frac{\mathrm{d} I}{\mathrm{~d} t}=\sigma E-\left(v_{4}+\mu+r_{I}\right) I, \\
& \frac{\mathrm{d} T}{\mathrm{~d} t}=v_{4} I-\left(\mu+r_{T}\right) T, \\
& \frac{\mathrm{d} R}{\mathrm{~d} t}=v_{3} E+r_{I} I+r_{T} T-\mu R,
\end{aligned}
$$

with initial densities

$$
\begin{gathered}
S(0)=S_{0} \geq 0, \\
V(0)=V_{0} \geq 0, \\
E(0)=E_{0} \geq 0, \\
I(0)=I_{0} \geq 0, \\
T(0)=T_{0} \geq 0, \\
R(0)=R_{0} \geq 0 .
\end{gathered}
$$

Let us denote by $X$ the vector of state variables, i.e., $X=$ $(S, V, E, I, T, R)^{T}$ with $X_{1}=S, X_{2}=V, X_{3}=E, X_{4}=I$, $X_{5}=T$, and $X_{6}=R$. Each equation in models (2)-(7) can be expressed as

$$
\frac{\mathrm{d} X_{i}}{\mathrm{~d} t}=g_{i}\left(X-\left\{X_{i}\right\}\right)-k_{i} X_{i}
$$

where $g_{i}$ are the functions and $k_{i}$ are the scalars for $i \in\{1,2, \ldots, 6\}$. Without loss of generality, from (9), we may have the following inequality:

$$
\frac{\mathrm{d} X_{i}}{\mathrm{~d} t} \geq-k_{i} X_{i} \Longleftrightarrow X_{i}(t) \geq X_{0} e^{-k_{i} t} .
$$

By the fact that $X_{0} \geq 0$ and $X_{0} e^{-k_{i} t}$ tend to zero as $t$ tends to infinity, all state variables remain nonnegative for $t \geq 0$, i.e., $X(t) \geq 0$. Furthermore, since $N(t)=S(t)+V(t)+$ $E(t)+I(t)+T(t)+R(t)$, we can obtain

$$
\frac{\mathrm{d} N}{\mathrm{~d} t}=\Lambda-\mu N \text {. }
$$

Ordinary differential equation (11) has the following explicit solution:

$$
N(t)=\frac{\Lambda}{\mu}+\left(N_{0}-\frac{\Lambda}{\mu}\right) e^{-\mu t},
$$

which tends to the steady-state solution $N(t)=a / \mu$ as $t$ tends to infinity. It means that the SVEITR models (2)-(7) is considered to be mathematically and epidemiologically well posed with bounded state variables. Thus, with respect to biological consideration, this system will be studied in the following positively invariant region:

$$
\mathbb{X}=\left\{(S, V, E, I, T, R)^{T} \in \mathbb{R}_{+}^{6} \mid S+V+E+I+T+R \leq \frac{a}{\mu}\right\} .
$$

3.3. Dynamical Properties. In this section, we discuss the dynamical properties of the SVEITR measles transmission model. We identify the existence of equilibrium points of the system and investigate their stability. We also provide the basic reproduction number of the model for analyzing the stability.

The point $X^{*} \in \mathbb{R}^{n}$ is an equilibrium point for the differential equation $\dot{X}=f(X)$ if $f\left(X^{*}\right)=0$ for all $t$. Thus, the 
equilibrium point refers to certain points in phase space with the property that if the system is placed in such a point, it stays there forever, because the derivatives of all the coordinates with respect to time are zero. All equilibrium points of systems (2)-(7) can be found by simultaneously solving the following nonlinear equations system:

$$
\begin{aligned}
\frac{\mathrm{d} S}{\mathrm{~d} t} & =0, \\
\frac{\mathrm{d} V}{\mathrm{~d} t} & =0, \\
\frac{\mathrm{d} E}{\mathrm{~d} t} & =0, \\
\frac{\mathrm{d} I}{\mathrm{~d} t} & =0, \\
\frac{\mathrm{d} T}{\mathrm{~d} t} & =0, \\
\frac{\mathrm{d} R}{\mathrm{~d} t} & =0 .
\end{aligned}
$$

In particular, a disease-free equilibrium point of the model is discovered by applying $E=I=T=R=0$. The disease-free equilibrium (DFE) of systems (2)-(7) is given by $P^{0}\left(S^{0}, V^{0}, 0,0,0,0\right)$, where

$$
\begin{aligned}
S^{0} & =\frac{\Lambda\left(\alpha\left(1-v_{2}\right)+\mu(1-p)\right)}{\mu\left(\alpha\left(1-v_{2}\right)+\left(v_{1}+\mu\right)\right)}, \\
V^{0} & =\frac{\Lambda\left(v_{1}+\mu p\right)}{\mu\left(\alpha\left(1-v_{2}\right)+\left(v_{1}+\mu\right)\right)} .
\end{aligned}
$$

In the presence of infection, systems (2)-(7) also have a nontrivial equilibrium point, known as an endemic equilibrium point (EEP). The endemic equilibrium of the SVEITR system is given by $P^{*}\left(S_{e}, V_{e}, E_{e}, I_{e}, T_{e}, R_{e}\right)$, where

$$
\begin{aligned}
S_{e} & =\frac{N_{e}\left((1-p) \Lambda-\alpha\left(1-v_{2}\right) V_{e}\right)}{\beta c_{I} I_{e}+\beta c_{T} T_{e}+N_{e}\left(v_{1}+\mu\right)} \\
V_{e} & =\frac{S_{e} v_{1}+p \Lambda}{\alpha\left(1-v_{2}\right)+\mu} \\
E_{e} & =\frac{\left(c_{I} I_{e}+c_{T} T_{e}\right) \beta S_{e}}{\left(v_{3}+\mu+\sigma\right) N_{e}}, \\
I_{e} & =\frac{\sigma E_{e}}{r_{I}+\mu+v_{4}}, \\
T_{e} & =\frac{v_{4} I_{e}}{r_{T}+\mu}, \\
R_{e} & =\frac{v_{3} E_{e}+r_{I} I_{e}+r_{T} T_{e}}{\mu} .
\end{aligned}
$$

The basic reproduction number is an important measure of transmissibility of the disease. It represents the expected number of secondary cases generated in a completely susceptible population, by a typical infected individual during its whole period of infectiousness. The stability of the disease-free equilibrium and the endemic equilibrium points can be analyzed using the basic reproduction number. The basic reproduction number can be calculated by using the next-generation operator approach of van den Driessche and Watmough [51]. The Jacobian matrices $F$ of new infection terms and $V$ of remaining transition terms evaluated using the variables $E, I, T$, and $R$ (in that order) are given, respectively, by

$$
\begin{aligned}
F & =\left[\begin{array}{ccc}
0 & \frac{\beta c_{I} S^{0}}{N} & \frac{\beta c_{T} S^{0}}{N} \\
0 & 0 & 0 \\
0 & 0 & 0
\end{array}\right], \\
V & =\left(\begin{array}{ccc}
v_{3}+\mu+\sigma & 0 & 0 \\
-\sigma & v_{4}+\mu+r_{I} & 0 \\
0 & -v_{4} & \mu+r_{T}
\end{array}\right) .
\end{aligned}
$$

The basic reproduction number $R_{0}$ is the spectral radius of $F V^{-1}$, i.e., $R_{0}=\rho\left(F V^{-1}\right)$. Thus, after a lengthy calculation, we may get

$$
R_{0}=\frac{\sigma \beta m_{1}\left(c_{I} m_{2}+c_{T} v_{4}\right)}{m_{2} m_{3} m_{4}},
$$

where

$$
\begin{aligned}
& m_{1}=\frac{\alpha\left(1-v_{2}\right)+\mu(1-p)}{\alpha\left(1-v_{2}\right)+v_{1}+\mu}, \\
& m_{2}=r_{T}+\mu, \\
& m_{3}=r_{I}+\mu+v_{4}, \\
& m_{4}=v_{3}+\mu+\sigma .
\end{aligned}
$$

The stability analyses of equilibrium points $P^{0}$ and $P^{*}$ are presented in the following theorems. The first theorem is proved by Routh-Hurwitz criterion, while the second theorem is proved based on bifurcation theory.

Theorem 1. If $R_{0}<1$, then the disease-free equilibrium $P^{0}$ is locally asymptotically stable, and if $R_{0}>1$, then it is unstable.

Proof. The Jacobian matrix at $P^{0}$ for systems (2)-(7) is given by 


$$
J^{0}=\left(\begin{array}{cccccc}
-v_{1}-\mu & \alpha\left(1-v_{2}\right) & 0 & -m_{1} \beta c_{I} & -m_{1} \beta c_{T} & 0 \\
v_{1} & -\alpha\left(1-v_{2}\right)-\mu & 0 & 0 & 0 & 0 \\
0 & 0 & -m_{4} & m_{1} \beta c_{I} & m_{1} \beta c_{T} & 0 \\
0 & 0 & \sigma & -m_{3} & 0 & 0 \\
0 & 0 & 0 & \tau & -m_{2} & 0 \\
0 & 0 & v_{3} & r_{I} & r_{T} & -\mu
\end{array}\right) .
$$

The characteristic equation of the matrix $J^{0}$, i.e., $\left|J^{0}-\omega I\right|=0$ for scalar $\omega$, is given by

$$
(\omega+\mu)^{2}\left(\omega+a_{1}\right)\left(\omega^{3}+b_{1} \omega^{2}+b_{2} \omega+b_{3}\right)=0,
$$

where

$$
\begin{aligned}
& a_{1}=\alpha\left(1-v_{2}\right)+v_{1}+\mu, \\
& b_{1}=m_{2}+m_{3}+m_{4}, \\
& b_{2}=m_{2} m_{3}+m_{2} m_{4}+\left(1-R_{0}\right) m_{3} m_{4}+\frac{m_{1} \sigma \beta c_{T} v_{4}}{m_{2}}, \\
& b_{3}=\left(1-R_{0}\right) m_{2} m_{3} m_{4} .
\end{aligned}
$$

From equation (21), we may have at most six eigenvalues with three of them negative, which are $\omega_{1}=\omega_{2}=-\mu<0$ and $\omega_{3}=-a_{1}<0$. However, $\omega_{4}, \omega_{5}$, and $\omega_{6}$ can be obtained by solving the following cubic equation:

$$
\omega^{3}+b_{1} \omega^{2}+b_{2} \omega+b_{3}=0
$$

Based on the Routh-Hurwitz criteria and by recalling that $\omega_{1}, \omega_{2}$, and $\omega_{3}$ are all negative, the equilibrium point $P^{0}$ is stable, if the following conditions are satisfied:

$$
b_{1}>0, b_{3}>0, b_{1} b_{2}>b_{3} \text {. }
$$

Because the values of all parameters are positive and $v_{2}, p \in[0,1]$, then $b_{1}>0$ and $b_{3}>0$ provided $R_{0}<1$. Next, we may obtain

$$
\begin{aligned}
b_{1} b_{2}= & \left(m_{2}+m_{3}+m_{4}\right)\left(m_{2} m_{3}+m_{2} m_{4}+\left(1-R_{0}\right) m_{3} m_{4}\right. \\
& \left.+\frac{m_{1} \sigma \beta c_{T} v_{4}}{m_{2}}\right)=\left(1-R_{0}\right) m_{2} m_{3} m_{4} \\
& + \text { positive terms }=b_{3}+\text { positive terms. }
\end{aligned}
$$

Then, $b_{1} b_{2}>b_{3}$, provided by $R_{0}<1$. These prove (24). As a result, the disease-free equilibrium $P^{0}$ for systems (2)-(7) is locally asymptotically stable if $R_{0}<1$ and unstable if $R_{0}>1$.
Theorem 2. If $R_{0}>1$, then the equilibrium $P^{*}$ is locally asymptotically stable and unstable if $R_{0}<1$.

Proof. Let us denote the right sides of systems (2)-(7) by the following functions:

$$
\begin{aligned}
& f_{1}(X):=(1-p) \Lambda+\left(1-v_{2}\right) \alpha X_{2}-\left(v_{1}+\lambda+\mu\right) X_{1} \\
& f_{2}(X):=p \Lambda-\left(\mu+\left(1-v_{2}\right) \alpha\right) X_{2}-v_{1} X_{1} \\
& f_{3}(X):=\lambda X_{1}-\left(v_{3}+\mu+\sigma\right) X_{3}, \\
& f_{4}(X):=\sigma X_{3}-\left(v_{4}+\mu+r_{I}\right) X_{4} \\
& f_{5}(X):=v_{4} X_{4}-\left(\mu+r_{T}\right) X_{5} \\
& f_{6}(X):=v_{3} X_{3}+r_{I} X_{4}+r_{T} X_{5}-\mu X_{6} .
\end{aligned}
$$

In a large variety of models, the dynamics of the models are influenced by the basic reproduction number $R_{0}$. It means that the models behave differently when $R_{0}<1$ and $R_{0}>1$. From (18), we can identify that the condition $R_{0}=1$ may be satisfied by

$$
\beta^{*}=\frac{m_{2} m_{3} m_{4}}{\sigma m_{1}\left(c_{I} m_{2}+c_{T} v_{4}\right)} .
$$

Thus, a sudden qualitative change of the system occurs when there is smooth change made to the value of $\beta$, from which we choose $\beta$ as the bifurcation parameter. Jacobian matrix (20) evaluated at the disease-free equilibrium $P^{0}$ with $\beta=\beta^{*}$ in (32), denoted by $J^{0}\left(\beta^{*}\right)$, has a simple zero eigenvalue with all other eigenvalues having a negative real part. By employing technique in Castillo-Chaves and Song [52], it can be shown that, for $R_{0}=1$, Jacobian matrix $J^{0}\left(\beta^{*}\right)$ has a right eigenvector which corresponds to the zero eigenvalue, given by $\left(u_{1}, u_{2}, u_{3}, u_{4}, u_{5}, u_{6}\right)^{T}$, where

$$
\begin{aligned}
& u_{1}=-\frac{m_{3} m_{4}\left(\alpha\left(1-v_{2}\right)+\mu\right) u_{4}}{\sigma \mu\left(\alpha\left(1-v_{2}\right)+v_{1}+\mu\right)} \\
& u_{2}=-\frac{m_{3} m_{4} v_{1} u_{4}}{\sigma \mu\left(\alpha\left(1-v_{2}\right)+v_{1}+\mu\right)}, \\
& u_{3}=\frac{m_{3} u_{4}}{\sigma}, \\
& u_{4}=\mu>0 \\
& u_{5}=\frac{v_{4} u_{4}}{m_{2}}, \\
& u_{6}=\left(r_{I}+\frac{m_{3} v_{3}}{\sigma}+\frac{r_{T} v_{4}}{m_{2}}\right) \frac{u_{4}}{\mu} .
\end{aligned}
$$


In the similar manner, the left eigenvector of $J^{0}\left(\beta^{*}\right)$, which corresponds to the zero eigenvalue, is given by $\left(w_{1}, w_{2}, w_{3}, w_{4}, w_{5}, w_{6}\right)^{T}$, where

$$
\begin{aligned}
& w_{1}=0, \\
& w_{2}=0, \\
& w_{3}=\sigma>0, \\
& w_{4}=\frac{m_{4} w_{3}}{\sigma}, \\
& w_{5}=\frac{c_{T} m_{3} m_{4} w_{3}}{\sigma\left(c_{I} m_{2}+c_{T} v_{4}\right)}, \\
& w_{6}=0 .
\end{aligned}
$$

Let us define the following quantities:

$$
\begin{aligned}
& a=\sum_{i, j, k=1}^{6} w_{k} u_{i} u_{j} \frac{\partial^{2} f_{k}\left(P^{0}, \beta^{*}\right)}{\partial X_{i} \partial X_{j}}, \\
& b=\sum_{i, j, k=1}^{6} w_{k} u_{i} \frac{\partial^{2} f_{k}\left(P^{0}, \beta^{*}\right)}{\partial X_{i} \partial \beta},
\end{aligned}
$$

where $f_{k}$ are given in (26)-(31). Calculation of all second partial derivatives of $f_{k}$ evaluated at $\left(P^{0}, \beta^{*}\right)$ provides

$$
\begin{aligned}
& a=\frac{u_{2} u_{4} w_{3} \beta^{*} \mu\left(\alpha\left(1-v_{2}\right)+\mu\right)}{a v_{1}}\left(c_{I}+\frac{v_{4}}{m_{2}}\right)<0, \\
& b=u_{4} w_{4} m_{1}\left(c_{I}+c_{T} v_{4}\right)>0 .
\end{aligned}
$$

Note that (37) holds because $u_{2}<0$. Since $a<0$ and $b>0$, then based on Castillo-Chaves-Song Theorem, we have the following conclusion regarding the stability of endemic equilibrium point $P^{*}$. The endemic equilibrium point $P^{*}$ is stable if $\beta>\beta^{*}$, otherwise unstable. By the fact that condition $\beta>\beta^{*}$ is equal to $R_{0}>1$, it is proved.

\section{Model with Optimal Control}

In an optimal control framework, it is assumed that the dynamics of state variable $X(t)$ can be controlled, at least partially, by a control variable $v(t)$. It means that the rate of change of $X(t)$ depends on time $t, X(t)$ itself, and control $v(t)$; i.e., the process is given by $\dot{X}=f(X, v, t)$. The problem addressed in an optimal control model is to characterize a control law for a given process within a period of time such that a certain performance criterion is achieved. In this section, the optimal control version of models (2)-(7) is studied, in which equipping the model with a set of control variables is imperative.

4.1. Optimal Control Model. Our primary objective of reformulating the SVEITR model into an optimal control setting is to examine the effect of immunization coverage and measles treatment in reducing the number of exposed and infected individuals as well as the cost implementation.
Therefore, for achieving this goal, we relax assumptions for several variables related to vaccination and treatment in the model with constant control. Instead of assigning the value to be constant, we represent proportion of susceptible individuals who receive the first dose of vaccine $v_{1}$, proportion of vaccinated individuals who obtain the second dose of vaccine $v_{2}$, the rate of measles therapy $v_{3}$, and the treatment rate for infected individuals $v_{4}$ as dynamic variables, i.e., $v_{i}=$ $v_{i}(t)$ for $i=1,2,3,4$. Control policies $v_{1}(t)$ and $v_{2}(t)$ are applied by administering measles vaccine by itself and in combinations such as the MMR (measles, mumps, rubella) vaccine to susceptible individuals in two doses, i.e., MCV1 and MCV2. Measles therapy $v_{3}(t)$ may be carried out by vitamin A supplementation to exposed individuals. Although there is no specific antiviral treatment for measles, we can consider policy $v_{4}(t)$ as supportive cares such as replacement fluids lost due to diarrhea and emesis, giving paracetamol or ibuprofen for the fever and prescribing an antibiotic for bacterial infection.

We make all control variables bounded, i.e.,

$$
\underline{v}_{i} \leq v_{i}(t) \leq \bar{v}_{i},
$$

for $i=1,2,3,4$ and for all $t \in[0, \bar{T}]$ with $\bar{T}$ as the control period. In (39), $\underline{v}_{i}$ and $\bar{v}_{i}$ constitute as the lower and upper bounds of the control effort. In most cases, we set $\underline{v}_{i}=0$ and $\bar{v}_{i}=1$ since $v_{i}(t)$ usually represents either a percentage or a proportion. If we set $\underline{v}_{i}=\bar{v}_{i}$, then it refers to the model with constant control discussed in the previous section. Let $v=$ $\left(v_{1}, v_{2}, v_{3}, v_{4}\right)^{T}$. The set of admissible control is therefore defined as

$\mathbb{V}=\left\{v \mid v_{i}(t)\right.$ is Lebesque measurable in $\left.[0, \bar{T}], v_{i}(t) \in\left[\underline{v}_{i}, \bar{v}_{i}\right], t \in[0, \bar{T}]\right\}$,

for $i=1,2,3,4$. The optimal control problem is a problem of finding a control law $v_{i}(t)$ among all admissible controls in $\mathbb{V}$ which brings systems (2)-(7) from the initial state (8) to a final state satisfying the terminal conditions such that it optimizes a cost functional. Note that for bounded Lebesgue measurable controls in (40) and nonnegative initial conditions (8), non-negative bounded solutions to systems (2)-(7) exist. We formulate the optimal control by considering a performance criterion given by

$$
\begin{aligned}
J(v):= & \int_{0}^{\bar{T}}\left(A_{1} E(t)+A_{2} I(t)+\frac{1}{2} B_{1} v_{1}^{2}(t)++\frac{1}{2} B_{2} v_{2}^{2}(t)\right. \\
& \left.+\frac{1}{2} B_{3} v_{3}^{2}(t)+\frac{1}{2} B_{4} v_{4}^{2}(t)\right) \mathrm{d} t
\end{aligned}
$$

subject to the state system given by (2)-(7), initial conditions (8), bounded controls (39), and terminal time conditions

$$
S(\bar{T}), V(\bar{T}), E(\bar{T}), I(\bar{T}), T(\bar{T}) \text {, and } R(\bar{T}) \text { are all free. }
$$

Criterion (41) adds up the number of exposed and infected individuals jointly with the control effort or the cost of implementing the control during the control period. In (41), the coefficients $A_{i}$ and $B_{i}$ are positive weights showing the 
relative importance among the terms. Therefore, the control objective is seeking the optimal control pair $v \in \mathbb{V}$ that minimizes the desired cost functional (41); i.e., we want to find $v^{*}$ such that

$$
J\left(v^{*}\right)=\min _{v \in \mathbb{V}} J(v) .
$$

Since the integrand of (41) is convex on the convex and closed admissible control set $\mathbb{V}$ in (40) and systems (2)-(7) are linear in the control variables $v_{i}$ as well as the state variables are bounded as in (13), then the existence of optimal control $v^{*}$ is guaranteed.

4.2. Optimality Conditions. We are now using Pontryagin's maximum principle [53] to derive the necessary conditions that a pair of optimal controls and corresponding state variables must satisfy. We begin by defining the Hamiltonian function that relates to system (2)-(7) as follows:

$$
\begin{aligned}
H(X, v, \omega):= & A_{1} E+A_{2} I+\frac{1}{2} B_{1} v_{1}^{2}+\frac{1}{2} B_{2} v_{2}^{2}+\frac{1}{2} B_{3} v_{3}^{2}+\frac{1}{2} B_{4} v_{4}^{2} \\
& +\omega_{1} f_{1}+\omega_{2} f_{2}+\omega_{3} f_{3}+\omega_{4} f_{4}+\omega_{5} f_{5}+\omega_{6} f_{6},
\end{aligned}
$$

where $\omega_{i}=\omega_{i}(t)$ for $i \in\{1,2, \ldots, 6\}$ are the adjoin functions and $f_{i}$ are the right-hand side of the equations system given in (26)-(31). Pontryagin's maximum principle consists of three blocks, namely, the dynamical systems obtained from $\dot{X}_{i}=H_{\omega_{i}}$ for $i \in\{1,2, \ldots, 6\}$, optimal controls derived from $H_{v_{i}}=0$ for $i \in\{1,2, \ldots, 6\}$, and the adjoin systems obtained from $\dot{\omega}_{i}=-H_{X_{i}}$ for $i \in\{1,2, \ldots, 6\}$. The first block is obviously systems (2)-(7) itself, which will produce the optimal state solutions $S^{*}, V^{*}, E^{*}, I^{*}, T^{*}$, and $R^{*}$. Other two blocks are stated in the following theorems.

Theorem 3. The optimal controls $v^{*}=\left(v_{1}^{*}, v_{2}^{*}, v_{3}^{*}, v_{4}^{*}\right)^{T}$ are given by

$$
\begin{aligned}
& v_{1}^{*}=\min \left\{\bar{v}_{1}, \max \left\{\underline{v}_{1}, \frac{\left(\omega_{1}-\omega_{2}\right) S}{B_{1}}\right\}\right\}, \\
& v_{2}^{*}=\min \left\{\bar{v}_{2}, \max \left\{\underline{v}_{2}, \frac{\left(\omega_{1}-\omega_{2}\right) \alpha V}{B_{2}}\right\}\right\}, \\
& v_{3}^{*}=\min \left\{\bar{v}_{3}, \max \left\{\underline{v}_{3}, \frac{\left(\omega_{3}-\omega_{6}\right) E}{B_{3}}\right\}\right\}, \\
& v_{4}^{*}=\min \left\{\bar{v}_{4}, \max \left\{\underline{v}_{4}, \frac{\left(\omega_{4}-\omega_{5}\right) I}{B_{4}}\right\}\right\} .
\end{aligned}
$$

Proof. By applying $H_{v_{i}}=0$ for $i \in\{1,2, \ldots, 6\}$, we have the following conditions:

$$
\begin{gathered}
B_{1} v_{1}-\left(\omega_{1}-\omega_{2}\right) S=0 \Longleftrightarrow v_{1}^{*}=\frac{\left(\omega_{1}-\omega_{2}\right) S}{B_{1}}, \\
B_{2} v_{2}-\left(\omega_{1}-\omega_{2}\right) \alpha V=0 \Longleftrightarrow v_{2}^{*}=\frac{\left(\omega_{1}-\omega_{2}\right) \alpha V}{B_{2}},
\end{gathered}
$$

$$
\begin{gathered}
B_{3} v_{3}-\left(\omega_{3}-\omega_{6}\right) E=0 \Longleftrightarrow v_{3}^{*}=\frac{\left(\omega_{3}-\omega_{6}\right) E}{B_{3}}, \\
B_{4} v_{4}-\left(\omega_{4}-\omega_{5}\right) I=0 \Longleftrightarrow v_{4}^{*}=\frac{\left(\omega_{4}-\omega_{5}\right) I}{B_{4}} .
\end{gathered}
$$

By considering bounded controls (39), we may express the above optimal controls as in (45)-(47).

Theorem 4. Given the optimal state solutions $S^{*}, V^{*}, E^{*}, I^{*}$, $T^{*}$, and $R^{*}$ associated with the optimal control pair $v^{*}$ given in (45)-(47), the adjoin variables $\omega_{i}(t)$ for $i \in\{1,2, \ldots, 6\}$ satisfy the following differential equation system:

$$
\begin{aligned}
& \dot{\omega}_{1}=\left(\omega_{1}-\omega_{3}\right) \lambda\left(1-\frac{S}{N}\right)+\left(\omega_{1}-\omega_{2}\right) v_{1}+\mu \omega_{1}, \\
& \dot{\omega}_{2}=\left(\omega_{3}-\omega_{1}\right) \lambda \frac{S}{N}+\left(\omega_{2}-\omega_{1}\right)\left(1-v_{2}\right) \alpha+\mu \omega_{2}, \\
& \dot{\omega}_{3}=-A_{1}+\left(\omega_{3}-\omega_{1}\right) \lambda \frac{S}{N}+\left(\omega_{3}-\omega_{4}\right) \sigma+\left(\omega_{3}-\omega_{6}\right) v_{3}+\mu \omega_{3},
\end{aligned}
$$

$$
\begin{aligned}
\dot{\omega}_{4}= & -A_{2}+\left(\omega_{1}-\omega_{3}\right)\left(\beta c_{I}-\lambda\right) \frac{S}{N}+\left(\omega_{4}-\omega_{5}\right) v_{4} \\
& +\left(\omega_{4}-\omega_{6}\right) r_{I}+\mu \omega_{4}, \\
\dot{\omega}_{5}= & \left(\omega_{1}-\omega_{3}\right)\left(\beta c_{T}-\lambda\right) \frac{S}{N}+\left(\omega_{5}-\omega_{6}\right) r_{T}+\mu \omega_{5}, \\
\dot{\omega}_{6}= & \left(\omega_{3}-\omega_{1}\right) \lambda \frac{S}{N}+\mu \omega_{6},
\end{aligned}
$$

with transversality conditions

$$
\begin{aligned}
& \omega_{1}(\bar{T})=0, \\
& \omega_{2}(\bar{T})=0, \\
& \omega_{3}(\bar{T})=0, \\
& \omega_{4}(\bar{T})=0, \\
& \omega_{5}(\bar{T})=0, \\
& \omega_{6}(\bar{T})=0 .
\end{aligned}
$$

Proof. When applying conditions $\dot{\omega}_{i}=-H_{X_{i}}$ for $i \in\{1,2, \ldots, 6\}$, recall that $\lambda$ is a function of all state variables as given in (1). Transversality conditions (58) emerge as we set that all state variables at terminal time are all free as stated in (42).

\section{Numerical Simulations}

In this section, we perform a numerical simulation to confirm dynamical properties of the system such as the stability of the equilibrium points, parameter sensitivity and bifurcation. For the model with optimal control, we investigate the effect of vaccination coverage, therapy, and treatment to the reduction of the number of exposed and 
infected individuals and the minimization of control effort. We consider a small community with a total population of 100 .

5.1. Stability, Parameter Sensitivity, and Bifurcation. Measles is a major public health problem in Indonesia. The government carried out additional measles immunization in August 2016 and Measles-Rubella (MR) immunization in Java from August to September 2017. The immunization campaign aims to provide additional immunity against measles so as to reduce the case of measles. This is evidenced by the decrease in cases and the absence of reports of outbreaks of measles in October 2017 to March 2018 in the immunization implementation area. We employ our proposed SVEITR model to simulate the data of measles cases reported in Indonesia. The parameter values used are sourced from the Directorate General of Disease Prevention and Control, Ministry of Health Republic of Indonesia, 2018.

Parameter values used in the simulation of model with constant control are presented in Table 2. In order to have the basic reproduction number $R_{0}$ located in different regions, we choose different values of the effective contact rate $\beta$ and the rate of exposed individuals become infective $\sigma$. For $\beta=0.03739$ and $\sigma=0.678$, we have $R_{0}=$ $0.1534<1$, and for $\beta=0.575$ and $\sigma=0.885$, we obtain $R_{0}=2.4238>1$.

In the case of $R_{0}<1$, it is discovered a disease-free equilibrium given by $P^{0}\left(S^{0}, V^{0}, 0,0,0,0\right)$, where $S^{0}=53.0733$ and $V^{0}=84.6078$. However, in the case of $R_{0}>1$, an endemic equilibrium point is found and is given by $\quad P^{*}\left(S^{*}, V^{*}, E^{*}, I^{*}, T^{*}, R^{*}\right)$, where $\quad S^{*}=21.8964$, $V^{*}=36.3463, E^{*}=0.5640, I^{*}=4.7753, T^{*}=1.6709$, and $R^{*}=72.4283$. In accordance with Theorem 1 , the diseasefree equilibrium is locally asymptotically stable. The state variable $X=(S, V, E, I, T, R)^{T}$ will tend to $P^{0}$ as $t$ tends to infinity. In Figure 2(a), it is shown that $E, I$, and $T$ are approaching zero at $t=60$ days. However, it needs longer time horizon to display the convergence of $S, V$, and $R$ to their equilibria. However, according to Theorem 2, the endemic equilibrium point is also locally asymptotically stable as described in Figure 2(b). In the former case, the measles disease could be extinct because the system is stable at a disease-free equilibrium point, and in the latter case, the disease could exist.

As a key threshold associates with the outbreaks, the basic reproduction number $R_{0}$ provides us with the estimate of the amount of effort that is required either to prevent epidemic or to remove infection from a population. According to (18), the basic reproduction number $R_{0}$ is affected by all parameters of the model, except $\chi$. Sensitivity analysis can then be applied to explore the impacts of fluctuations in parameters of the model on $R_{0}$ of greatest interest. In particular, sensitivity analysis can be used to determine parameters that exert the most influence to the value of $R_{0}$ when the disease spreads. In Hamby [54], the sensitivity index of $R_{0}$ with respect to parameter $q$, denoted by $\gamma_{q}$, can be calculated using the following formula:
TABLE 2: Parameter values for the model with constant control.

\begin{tabular}{lc}
\hline Parameter & Value \\
\hline$S(0)$ & 45 \\
$V(0)$ & 15 \\
$E(0)$ & 10 \\
$I(0)$ & 20 \\
$T(0)$ & 5 \\
$R(0)$ & 5 \\
$\wedge$ & 0.950 \\
$p$ & 0.250 \\
$\mu$ & 0.0069 \\
$\alpha$ & 0.100 \\
$\beta$ & $0.03739 ; 0.575$ \\
$c_{I}$ & 0.913 \\
$c_{T}$ & 0.978 \\
$\sigma$ & $0.678 ; 0.885$ \\
$r_{I}$ & 0.04762 \\
$r_{T}$ & 0.136 \\
$v_{1}$ & 0.150 \\
$v_{2}$ & 0.100 \\
$v_{3}$ & 0.080 \\
$v_{4}$ & 0.050 \\
\hline
\end{tabular}

$$
\gamma_{q}=\frac{\partial R_{0}}{\partial q} \frac{q}{R_{0}}
$$

In economics, formula (59) is known as elasticity, a measure of the proportional change of an economic variable in response to a change in the economic indicator. A positive sensitivity index indicates that an increase in the value of the parameter $q$ results in an increase in the basic reproduction number $R_{0}$. However, negative values of index indicate a decrease in the value of $R_{0}$ when the parameters increase. Table 3 provides the sensitivity index of parameters, calculated when $R_{0}<0$ and $R_{0}>1$. It is revealed that the effective contact rate $\beta$ contributes to the most influence on the basic reproductive number. If $\beta$ increases one unit, then $R_{0}$ also increases one unit.

5.2. Optimal Strategies. Numerical solutions of the optimal control problem presented in Section 4.1 are obtained by forwardly solving the dynamical systems (2)-(7) with initial conditions (8) and backwardly the adjoin system s(52)-(57) with terminal time conditions (58) using controls (45)-(47). The so-called forward-backward sweep method [55] and the well-known fourth-order Runge-Kutta algorithms were implemented to achieve the optimal solutions:

(i) Set an initial guess for the control variables $v_{i}^{0}(i=1,2,3,4)$

(ii) Solve forwardly-in-time state systems (2)-(7) with initial conditions (8) using the fourth-order Runge-Kutta scheme

(iii) Solve backwardly-in-time adjoin systems (52)-(57) with terminal conditions (58) using the fourth-order Runge-Kutta scheme

(iv) Calculate new controls (45)-(47) using the new values of the state and adjoin solutions and then 

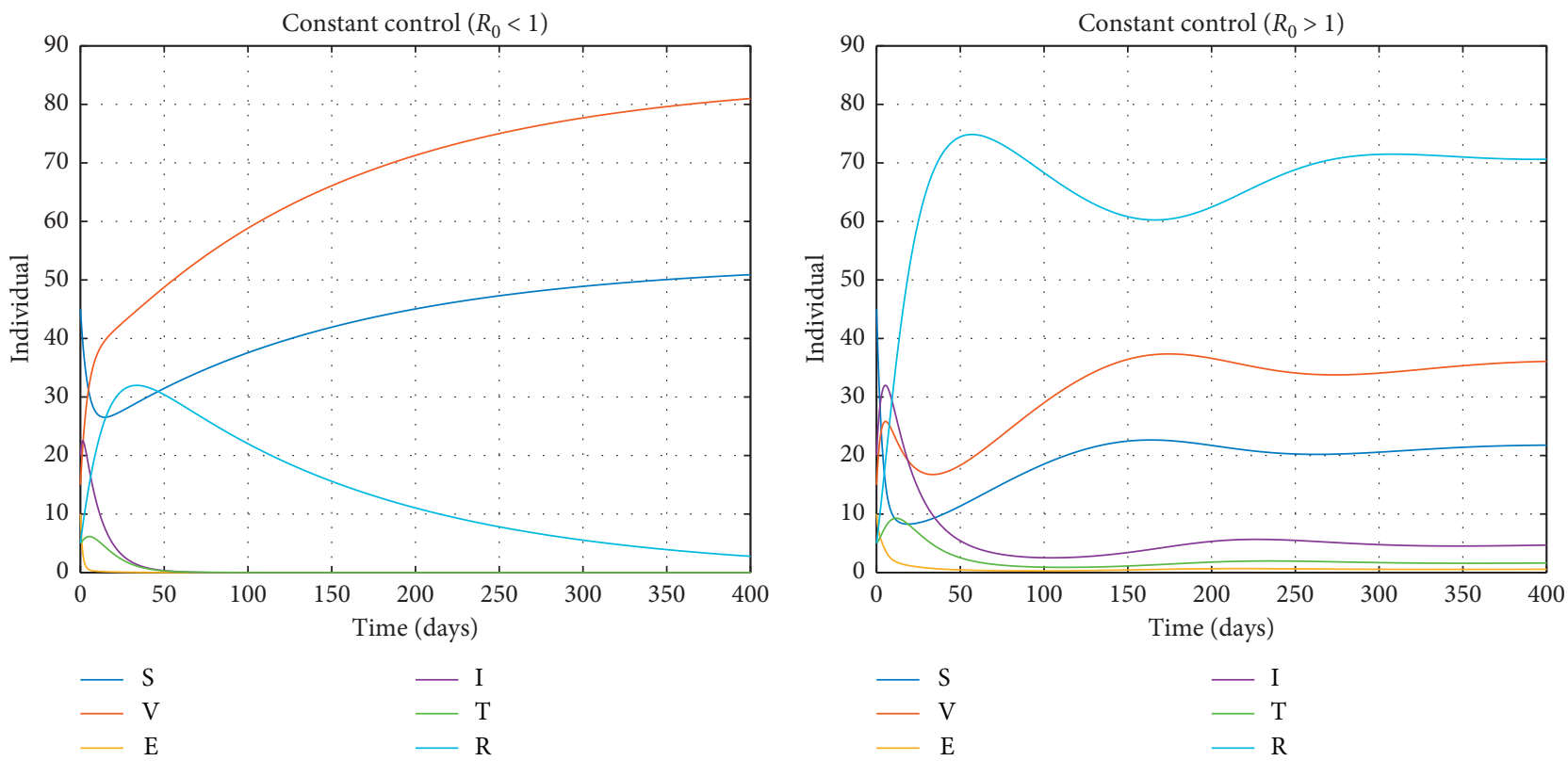

(a)

(b)

Figure 2: The dynamics of human population when (a) $R_{0}<1$ and (b) $R_{0}>1$.

TABLe 3: Parameter sensitivity index.

\begin{tabular}{lcc}
\hline Parameter & \multicolumn{2}{c}{ Sensitivity index } \\
\hline$\wedge$ & $R_{0}<1$ & $R_{0}>1$ \\
$p$ & 0.0000 & 0.0000 \\
$\mu$ & -0.0181 & -0.0181 \\
$\alpha$ & -0.1223 & -0.1204 \\
$\beta$ & 0.5811 & 0.5811 \\
$c_{I}$ & 1.0000 & 1.0000 \\
$c_{T}$ & 0.7274 & 0.7274 \\
$\sigma$ & 0.2726 & 0.2726 \\
$r_{I}$ & 0.1136 & 0.0894 \\
$r_{T}$ & -0.6075 & -0.6075 \\
$v_{1}$ & -0.0646 & -0.0646 \\
$v_{2}$ & -0.1046 & -0.0823 \\
$v_{3}$ & 0.2726 & 0.2726 \\
$v_{4}$ & -0.8734 & -0.8734 \\
\hline
\end{tabular}

update the controls by averaging old and new controls

(v) Iterate the process until the solutions converge within sufficiently small level of tolerance

Values of parameters in Table 4 were used in the simulation of the model with optimal control. Most of the parameters in Table 1 remain unchanged in values. We only change the value of $\alpha, \beta$, and $\sigma$ and treat $v_{i}$ as now bounded dynamic variables rather than constants. We also introduce parameters $A_{i}$ and $B_{j}$ as weights of performance criterion (41). We set more priority to the minimization of the number of exposed and infected individuals and assign less priority to the treatment cost.
TABLe 4: Parameter values for the model with optimal control.

\begin{tabular}{lc}
\hline Parameter & Value \\
\hline$S(0)$ & 45 \\
$V(0)$ & 15 \\
$E(0)$ & 10 \\
$I(0)$ & 20 \\
$T(0)$ & 5 \\
$R(0)$ & 5 \\
$\wedge$ & 0.950 \\
$p$ & 0.250 \\
$\mu$ & 0.0069 \\
$\alpha$ & 0.221 \\
$\beta$ & 0.678 \\
$c_{I}$ & 0.913 \\
$c_{T}$ & 0.978 \\
$\sigma$ & 0.265 \\
$r_{I}$ & 0.143 \\
$r_{T}$ & 0.136 \\
$A_{1}$ & 0.200 \\
$A_{2}$ & 0.200 \\
$B_{1}$ & 0.150 \\
$B_{2}$ & 0.150 \\
$B_{3}$ & 0.150 \\
$B_{4}$ & 0.050 \\
\hline Variable & Range \\
\hline$v_{1}(t)$ & {$[0,0.86]$} \\
$v_{2}(t)$ & {$[0,0.69]$} \\
$v_{3}(t)$ & {$[0,0.40]$} \\
$v_{4}(t)$ & {$[0,0.50]$} \\
\hline &
\end{tabular}

In the first simulation, we compare the dynamic of measles transmission under constant and optimal controls. The model with constant control was run by setting all 


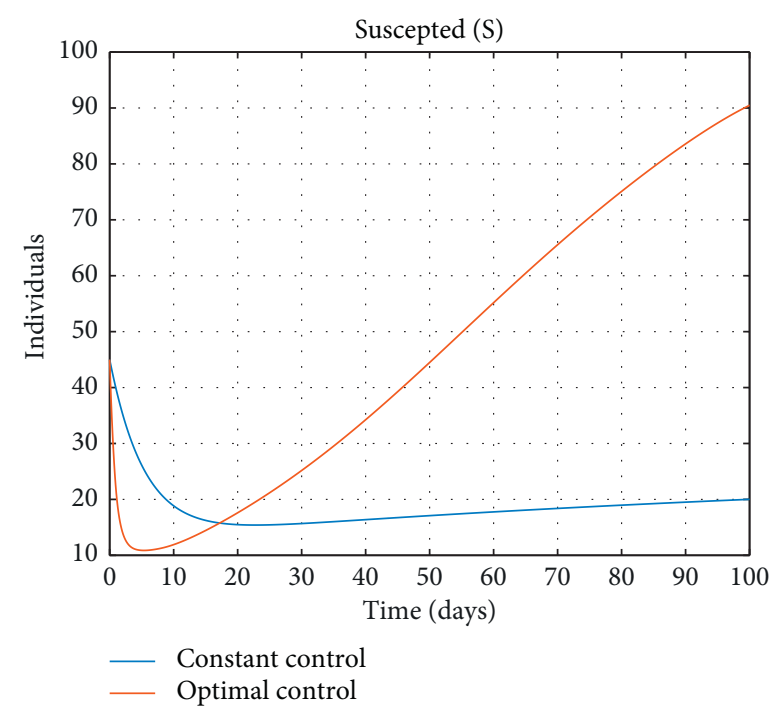

(a)

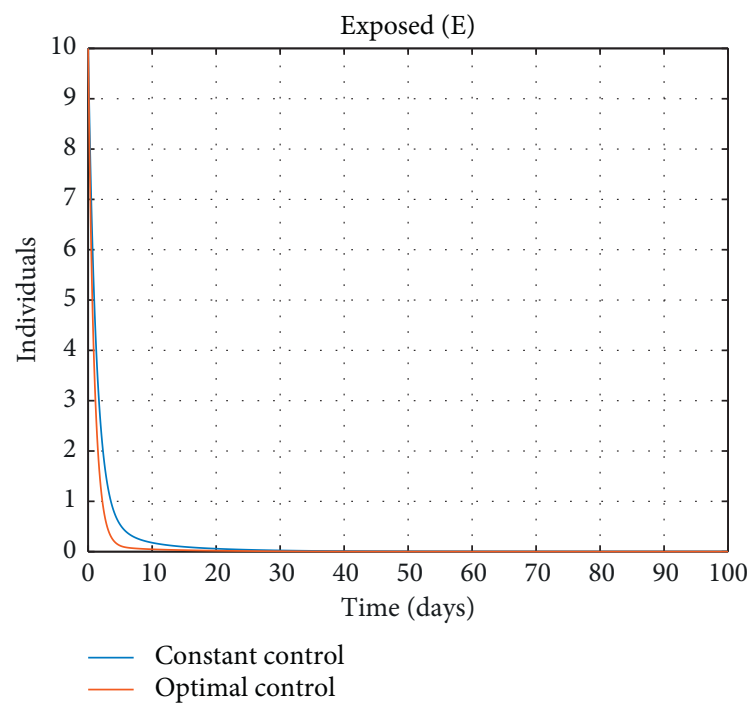

(c)

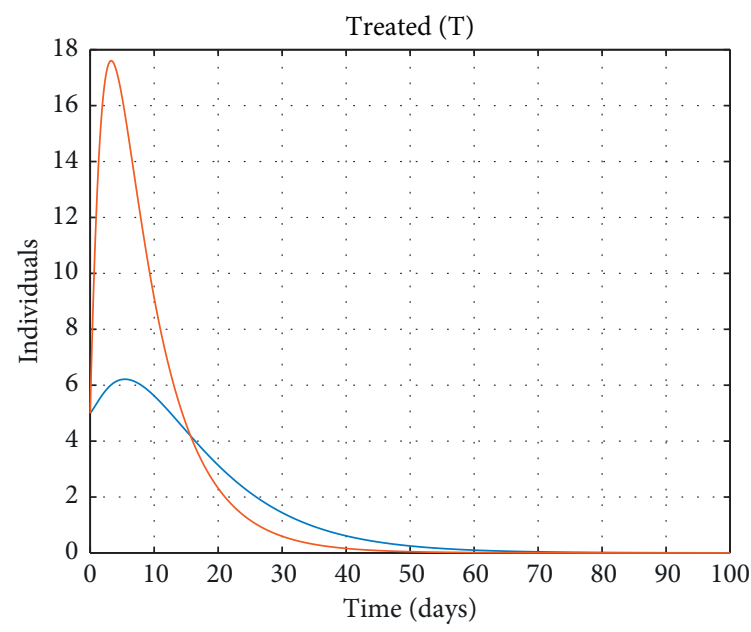

Constant control Optimal control

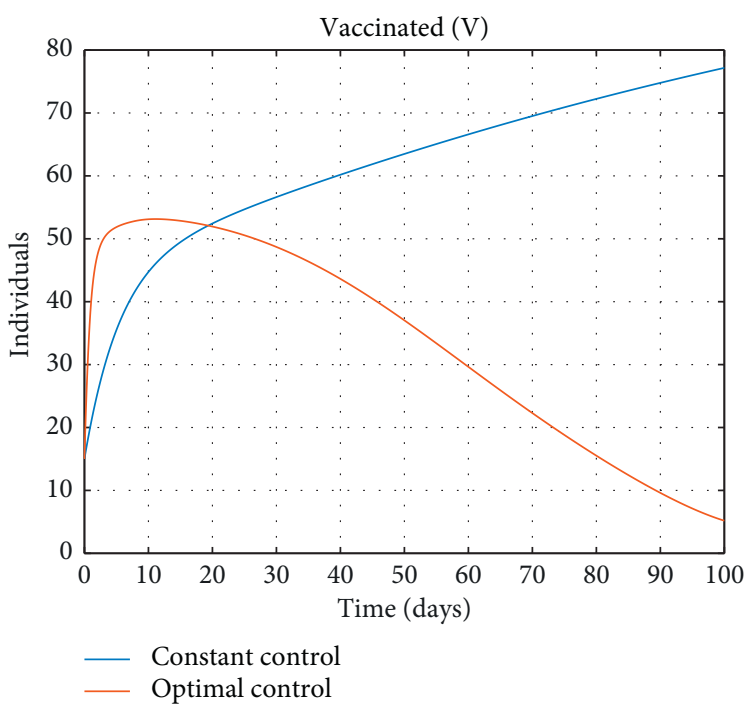

(b)

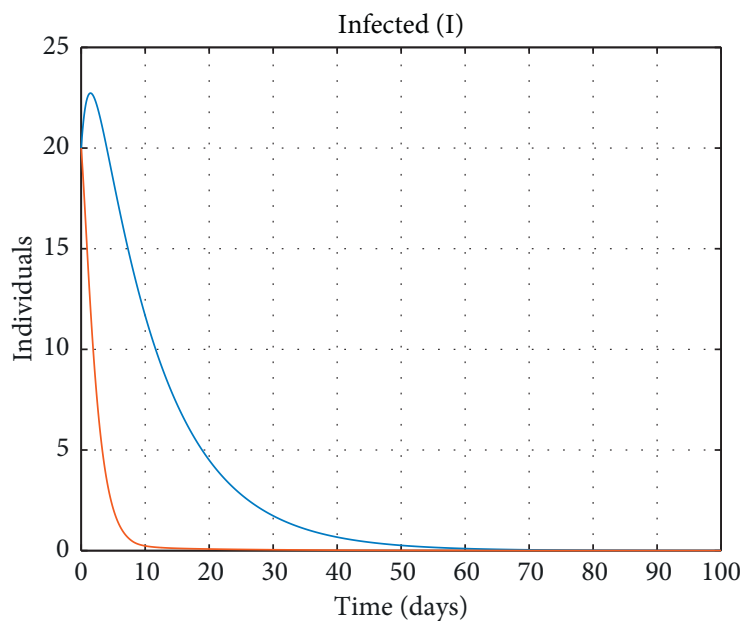

- Constant contro Optimal control

(d)

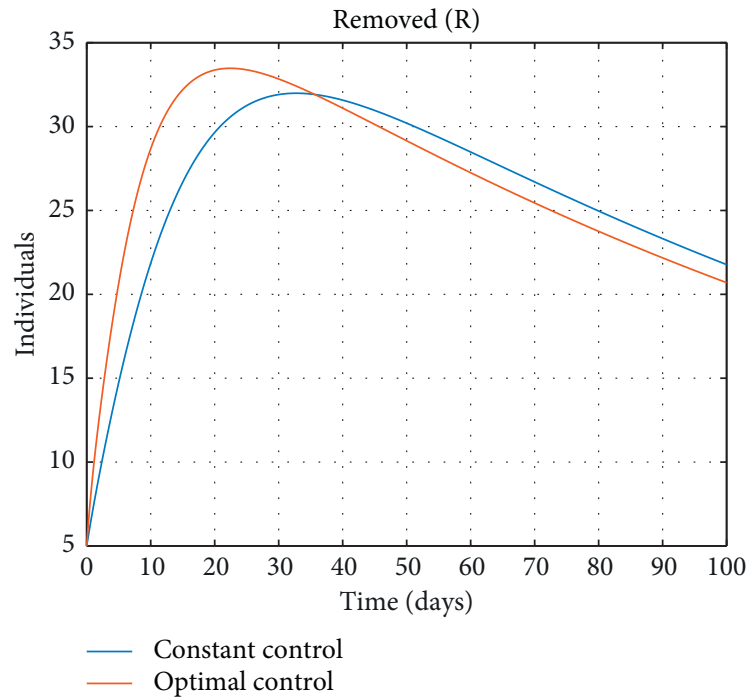

(f)

Figure 3: The state variables with constant and optimal controls. 


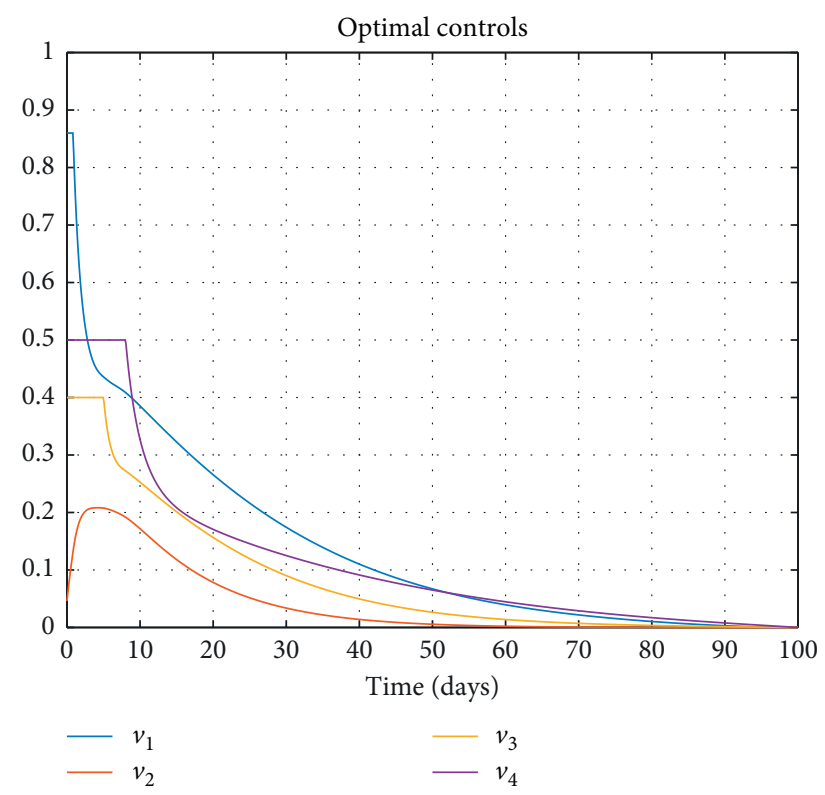

(a)
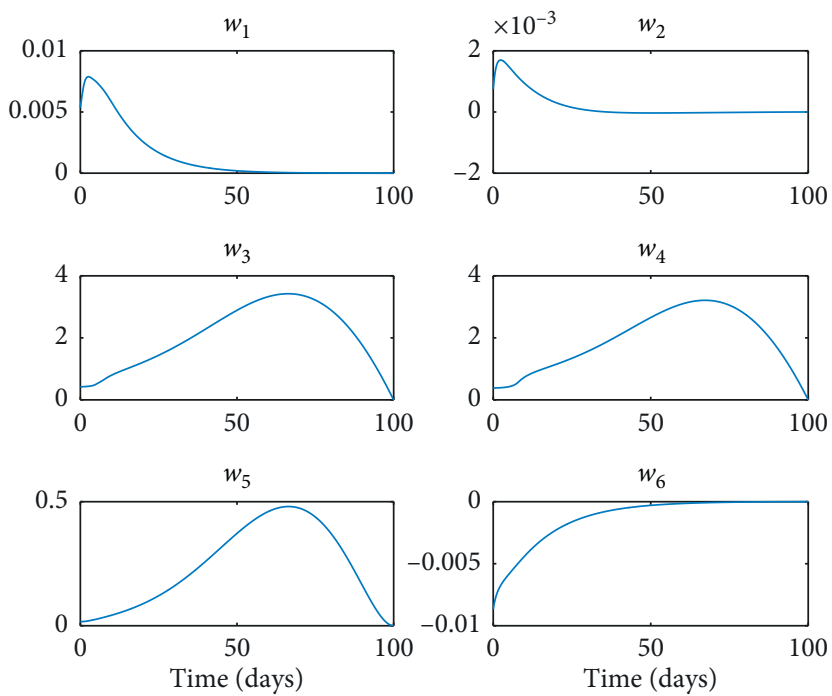

Figure 4: (a) Optimal controls and (b) adjoin variables.

TABle 5: Control scenarios.

\begin{tabular}{|c|c|c|c|c|}
\hline \multirow{2}{*}{ Policy } & \multirow{2}{*}{ Variable } & \multicolumn{3}{|c|}{ Coverage } \\
\hline & & Low (\%) & Moderate (\%) & High (\%) \\
\hline First dose vaccine & $v_{1}$ & 60 & 86 & 95 \\
\hline Second dose vaccine & $v_{2}$ & 30 & 69 & 75 \\
\hline Therapy & $v_{3}$ & 20 & 40 & 50 \\
\hline Treatment & $v_{4}$ & 15 & 50 & 60 \\
\hline
\end{tabular}

control variables constant for all time, i.e., $v_{1}=0.15$, $v_{2}=0.10, v_{3}=0.08$ and $v_{4}=0.05$. However, the model with optimal control was executed by letting optimization mechanism select the best control variables among admissible controls in $\mathbb{V}(40)$. We specified the bounded controls as follows: $\quad v_{1}(t) \in[0,0.86], \quad v_{2}(t) \in[0,0.69]$, $v_{3}(t) \in[0,0.40]$, and $v_{4}(t)=[0,0.50]$. The upper bounds for $v_{1}(t)$ and $v_{2}(t)$ were defined based on WHO estimation that only $86 \%$ children have received the first dose of measles vaccine and $69 \%$ the second in 2019.

Figure 3 illustrates the dynamic of state variables under constant control (blue line) and optimal controls (red line). Figure 3(b) depicts the dynamics of population in vaccinated class. In the early period of control, the number of vaccinated individuals grows quickly due to the vaccine application. This transfers most of the susceptible individuals to vaccinated compartment (Figure 3(a)). The number of vaccinated individuals began to decrease on day 20 , which increases the number of susceptibles. Figures 3(c) and 3(d) show the effect of measles therapy on exposed individuals and the effect of treatment on infected individuals. It seems that therapy and treatment effectively reduce the number of exposed and infected people. Figure 3(e) informs that the treatment for measles was mostly provided in the first five days of control period. Even though the constant control policy successfully decreased the number of exposed and infected individuals, the optimal control policy completed the same task even better. These facts, however, confirm that vaccine is very effective at preventing measles.

Figure 4(a) describes how the optimal policies $v_{i}(i=$ $1,2,3,4)$ should be implemented. It is suggested that the first-dose vaccine should be administered in full capacity, i.e., with $86 \%$ of coverage, during the first five days. The coverage was then decreased rather quickly until the end of control period. We should apply the second dose vaccine in full capacity a bit longer, i.e., with $69 \%$ of coverage for the first twenty days and then rapidly reduce the intensity. At the same time, full capacity of measles therapy and treatment should be performed during the first fifteen and eight days, respectively. In contrast, constant control policies must maintain the level of coverage constant all the time. However, in optimal control policies, we optimally adjust the level of coverage such that it minimizes the performance criterion. Figure 4(b) presents the dynamic of adjoin variables $\omega_{i}(i=1,2, \ldots, 6)$. It is confirmed that the terminal time conditions $\omega_{i}(\bar{T})=0(i=1,2, \ldots, 6)$ in (58) were satisfied.

To assess in more detail the effect of coverage of vaccination, measles therapy, and treatment, we develop three scenarios based on their levels of intensity: low, moderate, 

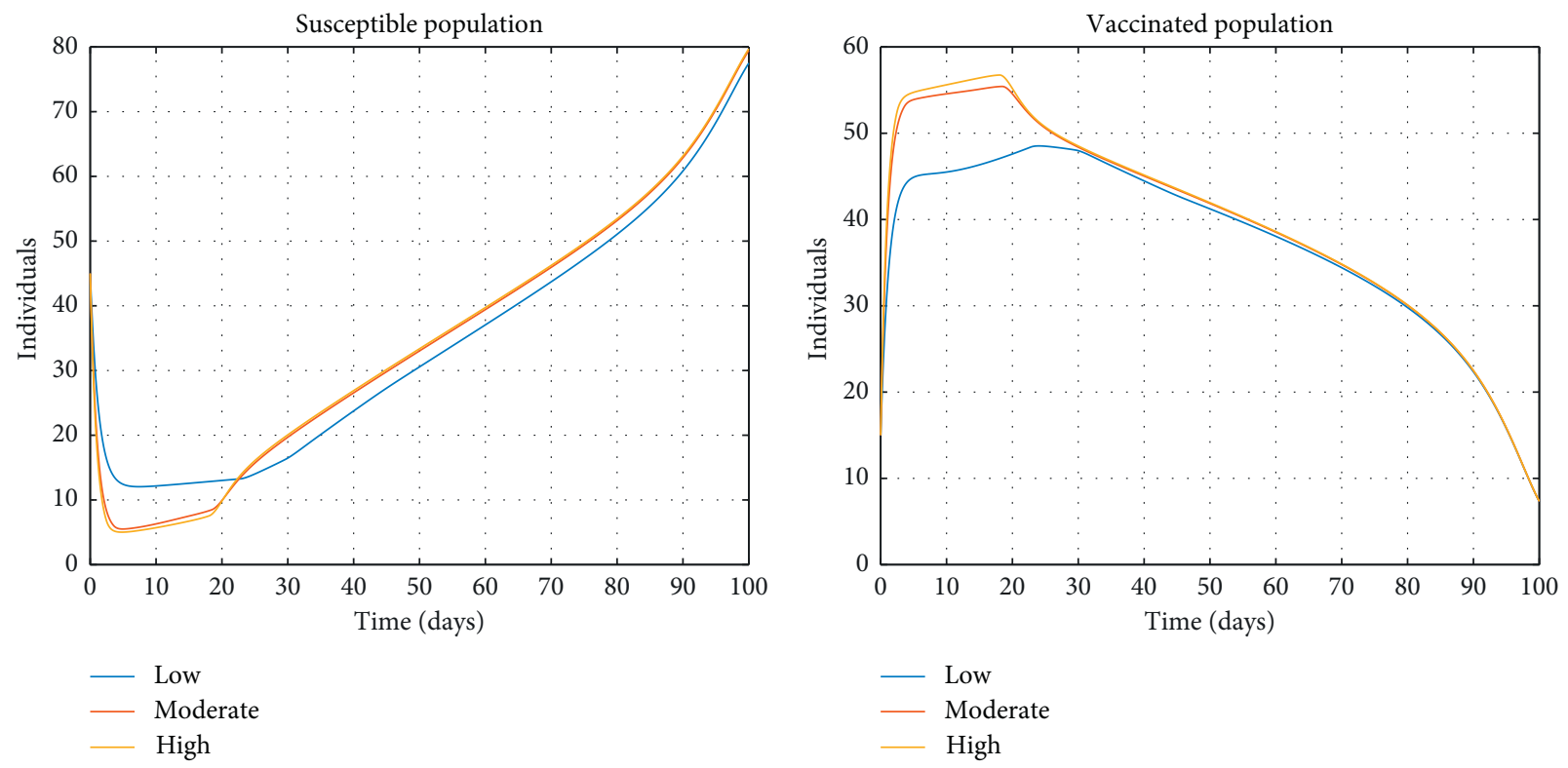

(a)

(b)
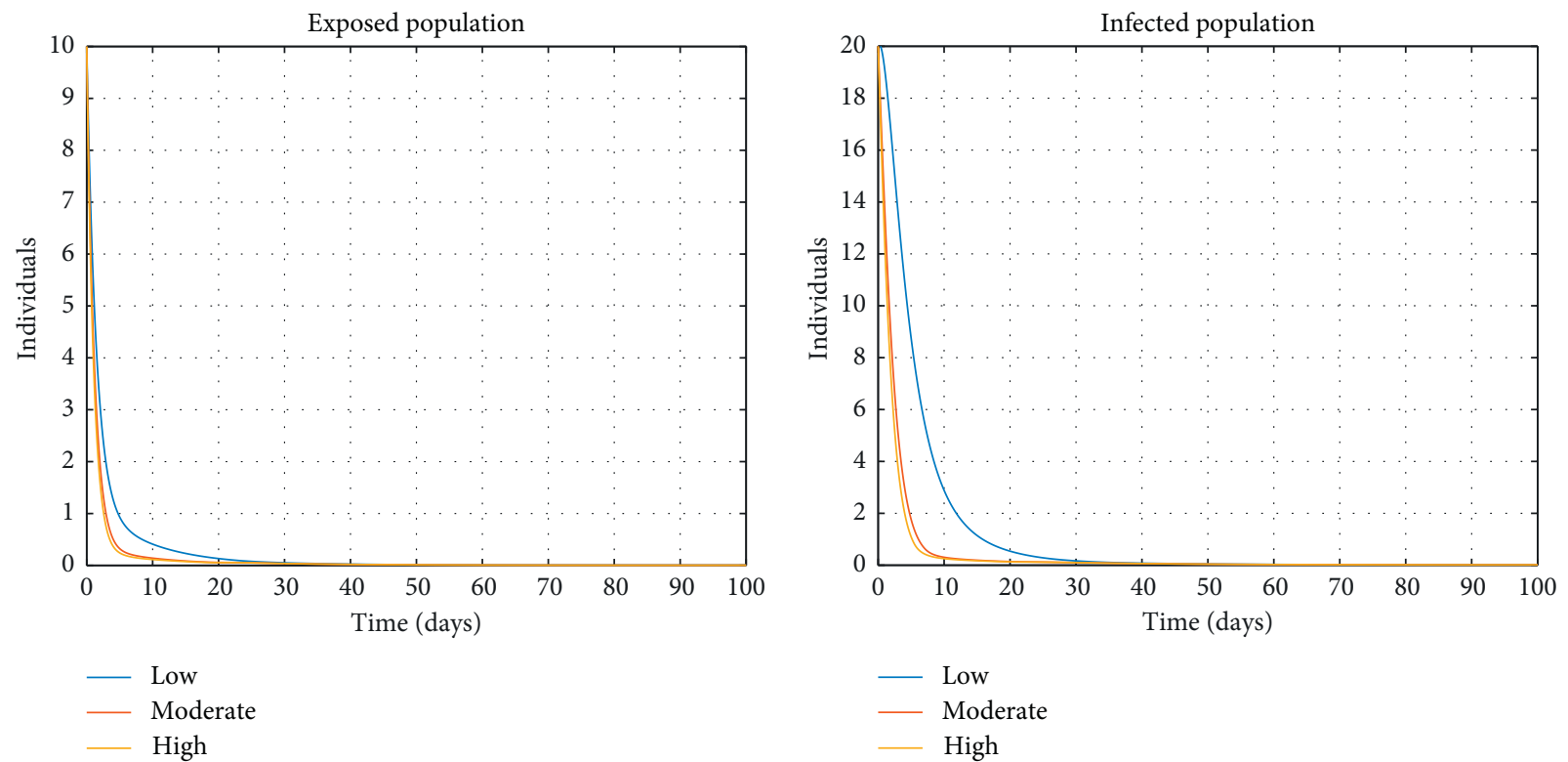

(c)

(d)

Figure 5: Continued. 


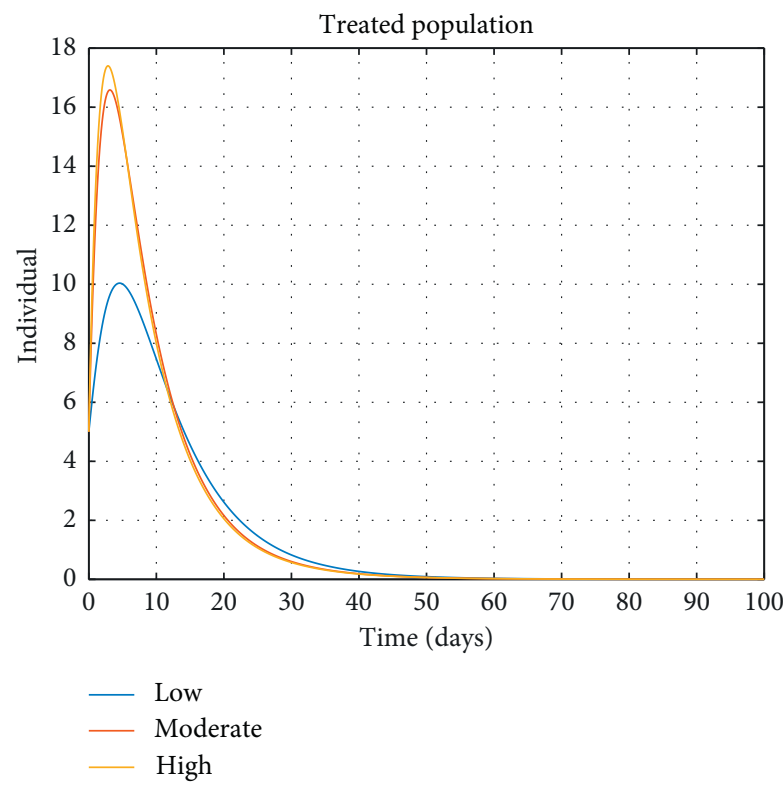

(e)

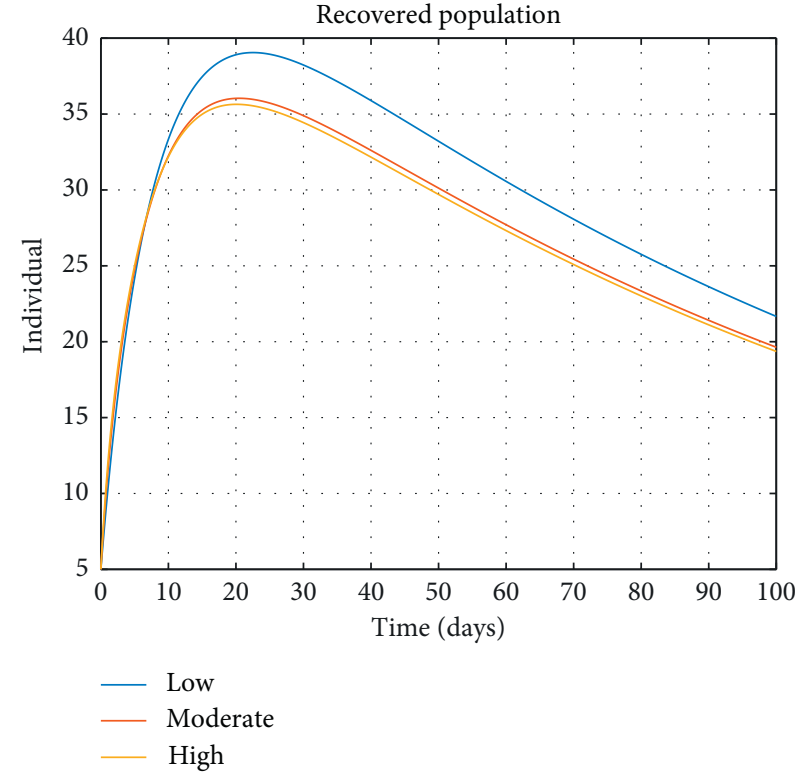

(f)

FIGURE 5: The effect of vaccination coverage, therapy and treatment under low, moderate and high scenarios.

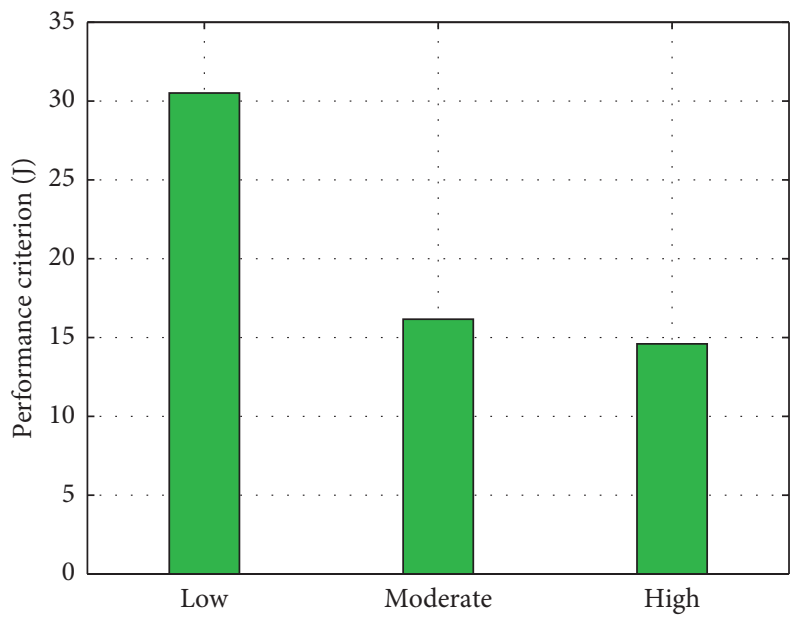

FIgURE 6: Performance criterion.

and high as described in Table 5 . We consider the existing vaccination coverage of $86 \%$ for MCV1 and $69 \%$ for MCV2 as the baseline (moderate scenario) and set the 2020 target coverage of $95 \%$ for MCV1 and $75 \%$ for MCI2 as the high scenario. We also take into consideration a situation where only small coverages were realized, i.e., the low scenario.

Figure 5 illustrates the population dynamics in each class under three different scenarios. It is shown that all scenarios produce similar shape of curves. By expanding the coverage of vaccination, therapy, and treatment, the number of vaccinated individuals increases and those of exposed and infected individuals decrease. It is also confirmed that moderate and high scenarios show better performance in reducing the number of exposed and infected individuals than low scenario, despite only a few differences on the results between moderate and high scenarios. From Figure 6, it can be calculated that moderate and high scenarios, respectively, reduce the cost functional (41) up to $47 \%$ and $52 \%$ of that achieved by low scenario. Meanwhile, there was only a cost reduction of no more than $10 \%$ by high scenario against moderate scenario.

The optimal controls under low, moderate, and high scenarios are depicted in Figure 7. All control policies share similarities in implementation, i.e., control policies under moderate and high scenarios were accomplished at the maximum rate during the first few days of control period. However, policies under low scenario need longer time. In the second half of the control period, i.e., after day fifty, controls under all scenarios were approximately applied at the same rate. 


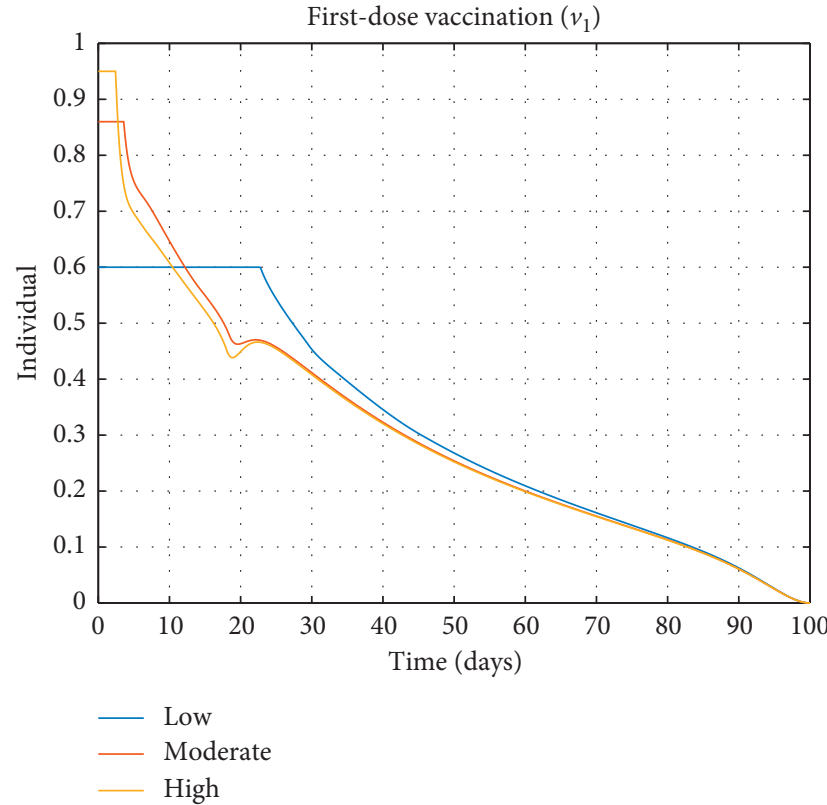

(a)

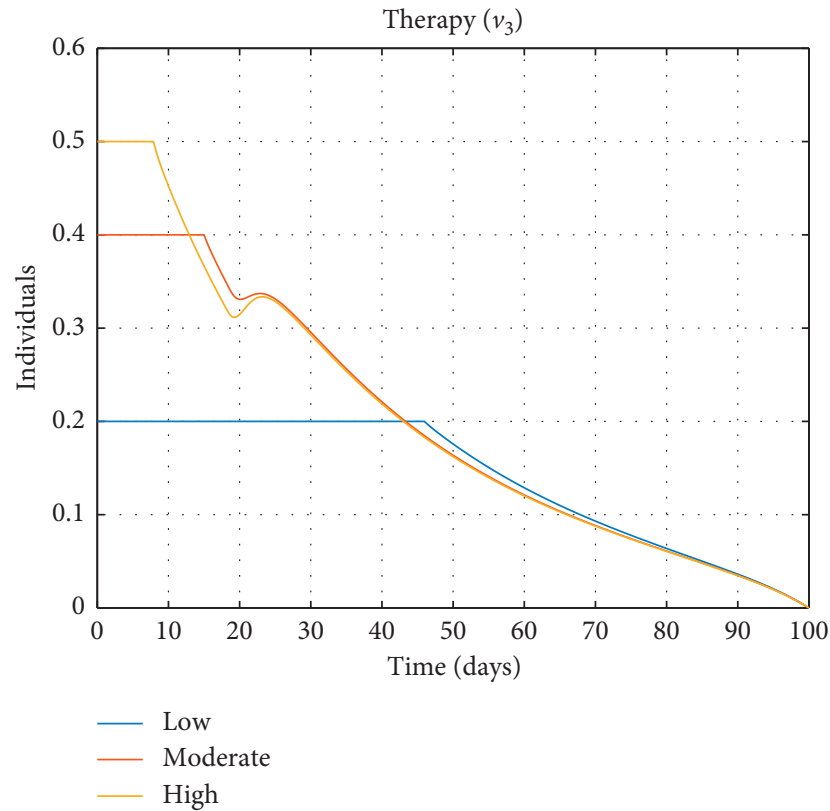

(c)

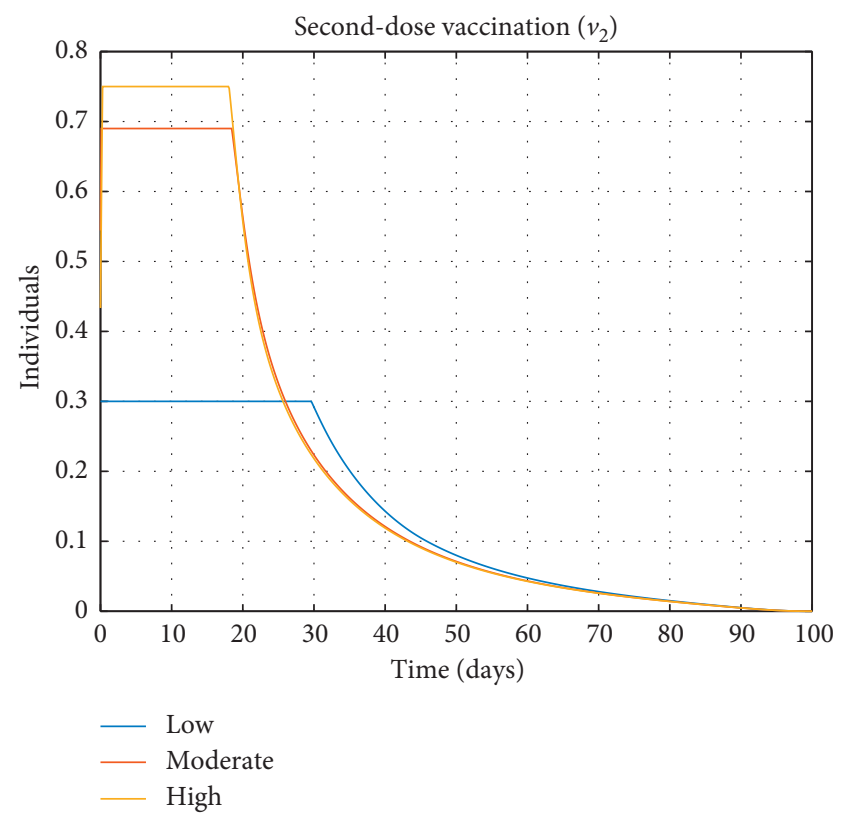

(b)

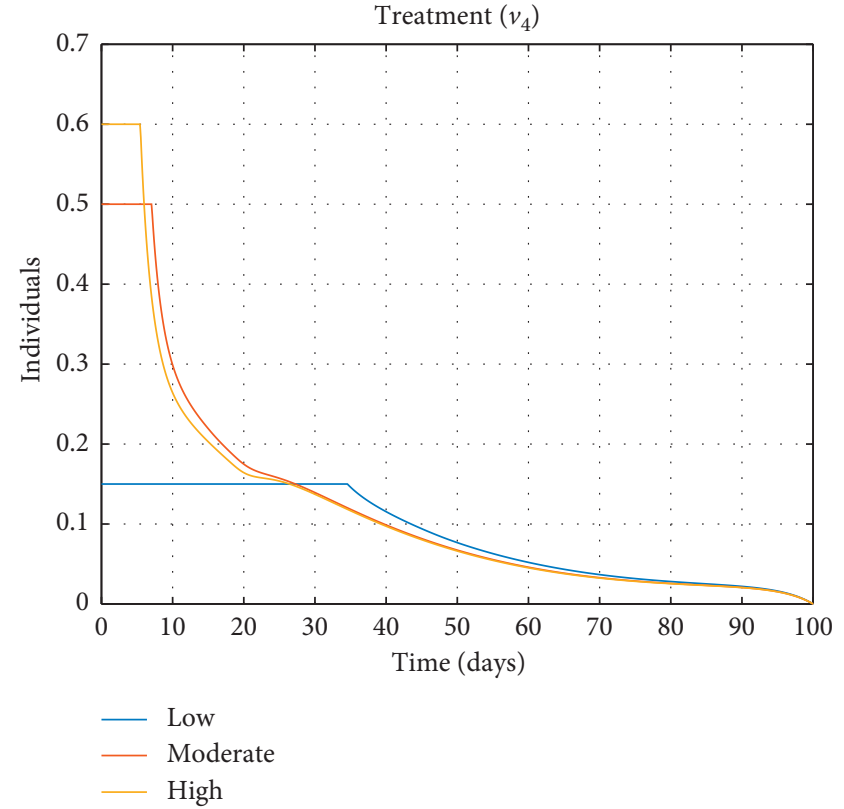

(d)

Figure 7: The optimal controls using different scenarios.

\section{Concluding Remarks}

An autonomous nonlinear differential equation system for measles dynamics, which incorporates constant vaccination, therapy, and treatment rates, is considered first of all. The SVEITR model consists of six compartments (susceptible, vaccinated, exposed, infected, treated, and recovered) to categorize individuals according to their disease state. The stability analysis of the model is carried out towards diseasefree and endemic equilibrium points using Routh-Hurwitz criteria and bifurcation theory. It is shown that the stability of the equilibrium points depends on the basic reproduction number $\left(R_{0}\right)$. If $R_{0}<1$, the disease-free equilibrium point will be stable, otherwise unstable. The endemic equilibrium point is in an asymptotically stable condition if $R_{0}>1$, otherwise unstable. Sensitivity analysis of the model reveals that the effective contact rate is the most influencing parameter to the model.

The model with constant controls is then extended to include time-dependent vaccination, therapy, and treatment rates, resulting in the model with optimal controls. The Pontryagin maximum principle is employed to derive the necessary conditions for existence of an optimal control pair that minimizes the number of exposed and infected 
individuals jointly with the control effort. Numerical simulations constructed from the forward-backward sweep method and the fourth-order Runge-Kutta algorithm are performed to evaluate three scenarios based on vaccination, therapy, and treatment coverages. It is demonstrated that moderate and high levels of coverage can effectively reduce the measles cases.

In reality, infections caused by more than one disease can circulate within the same period. Changes in dynamics that develop in one disease affect the transmission dynamics of other diseases. Cases that frequently occur are HIV/AIDS infection coinciding with TB infection or between malaria and cholera. Study on interconnection between two diseases as a coinfection offers many advantages ([56, 57]). In situation of acute complications, measles infection can also arise simultaneously with pneumonia infection [58]. Thus, development of a measles-associated pneumonia model is very sensible in understanding the parasite interaction, disease transmission and progression, and establishment of prevention and control strategies. Measles is a contagious disease that can spread rapidly to several areas [59]. In this direction, development of a multiregional measles transmission model is a necessity in understanding the diffusion of measles disease across a landscape, as did by Athina et al. [60] for two regional cases of malaria.

\section{Data Availability}

All data used for this study are publicly available. No new data were created during this study.

\section{Conflicts of Interest}

The authors declare that there are no conflicts of interest regarding the publication of this paper.

\section{References}

[1] S. Soucheray, Global Measles Outbreaks Make 2019 a RecordSetting Year, CIDRAP News, Minneapolis, MN, USA, 2019.

[2] World Health Organization (WHO), Measles, World Health Organization (WHO), Geneva, Switzerland, 2019, https:// www.who.int/news-room/fact-sheets/detail/measles.

[3] Centers for Disease Control and Prevention (CDC), Epidemiology and Prevention of Vaccine-Preventable Diseases, J. Hamborsky, A. Kroger, and S. Wolfe, Eds., Public Health Foundation, Washington, DC, USA, 13th edition, 2015.

[4] A. A. Gershon, "Measles virus (rubeola)," in Mandell, Douglas, and Bennett's Infectious Disease Essentials, J. E. Bennett, R. Dolin, and M. J. Blaser, Eds., Elsevier, Philadelphia, PA, USA, pp. 220-221, 2017.

[5] World Health Organization (WHO), Global Measles and Rubella Strategic Plan: 2012-2020, WHO Press, Geneva, Switzerland, 2012.

[6] D. Barne and D. Wadhwa, Measles Vaccine Coverage at 85\% Globally, World Bank Blogs, Washington, DC, USA, 2019.

[7] K. Manakongtreecheep and R. Davis, "A review of measles control in Kenya, with focus on recent innovations," Pan African Medical Journal, vol. 27, no. 3, pp. 1-7, 2017.

[8] S. Sharma, WHO South-East Asia Region Sets 2023 Target to Eliminate Measles, Rubella, Press Release WHO South-East Asia Regional Office, Geneva, Switzerland, 2019.
[9] WHO/Europe, Vaccination against Measles Increases amid Ongoing Measles Outbreaks in Europe, Press Release WHO Regional Office for Europe, Europe, 2019.

[10] PAHO/WHO, Epidemiological Update: Measles, Pan American Health Organization/World Health Organization, Washington, DC, USA, 2019.

[11] P. Yan and G. Chowell, Quantitative Methods for Investigating Infectious Disease Outbreaks, Springer, Cham, Switzerland, 2019.

[12] E. Njeuhmeli, M. Schnure, A. Vazzano et al., "Using mathematical modeling to inform health policy: a case study from voluntary medical male circumcision scale-up in eastern and southern Africa and proposed framework for success," PLoS One, vol. 14, no. 3, Article ID e0213605, 2019.

[13] K. G. Kristinsson, "Mathematical models as tools for evaluating the effectiveness of interventions: a comment on Levin," Clinical Infectious Diseases, vol. 33, no. s3, pp. S174-S179, 2001.

[14] E. A. Bakare, Y. A. Adekunle, and K. O. Kadiri, "Modelling and simulation of the dynamics of the transmission of measles," International Journal of Computer Trends and Technology, vol. 3, no. 1, pp. 174-178, 2012.

[15] G. Bolarian, "On the dynamical analysis of a new model for measles infection," International Journal of Mathematics Trends and Technology, vol. 7, no. 2, pp. 144-155, 2014.

[16] A. A. Momoh, M. O. Ibrahim, I. J. Uwanta, and S. B. Manga, "Mathematical model for control of measles epidemiology," International Journal of Pure and Applied Mathematics, vol. 87, no. 5, pp. 707-717, 2013.

[17] A. A. Momoh, M. O. Ibrahim, I. J. Uwanta, and S. B. Manga, "Modelling the effect of vaccination on the transmission dynamics of measles," International Journal of Pure and Applied Mathematics, vol. 88, no. 3, pp. 381-390, 2013.

[18] M. O. Fred, J. K. Sigey, J. A. Okello, J. M. Okwyo, and G. J. Kang'ethe, "Mathematical modelling on the control of measles by vaccination: case study of KISII county, Kenya," The SIJ Transactions on Computer Science Engineering and Its Applications (CSEA), vol. 2, no. 4, pp. 38-46, 2014.

[19] O. O. Onyejekwe and E. Z. Kebede, "Epidemiological modeling of measles infection with optimal control of vaccination and supportive treatment," Applied and Computational Mathematics, vol. 4, no. 4, pp. 264-274, 2015.

[20] N. Thakkar, S. S. A. Gilani, Q. Hasan, and K. A. McCarthy, "Decreasing measles burden by optimizing campaign timing," Proceedings of the National Academy of Sciences, vol. 116, no. 22, pp. 11069-11073, 2019.

[21] A. McKee, K. Shea, and M. J. Ferrari, "Optimal vaccine schedules to maintain measles elimination with a two-dose routine policy," Epidemiology and Infection, vol. 145, no. 2, pp. 227-235, 2016.

[22] L. Pang, S. Ruan, S. Liu, Z. Zhao, and X. Zhang, "Transmission dynamics and optimal control of measles epidemics," Applied Mathematics and Computation, vol. 256, pp. 131-147, 2015.

[23] L. K. Beay, "Modelling the effects of treatment and quarantine on measles," AIP Conference Proceedings, vol. 1937, Article ID 020004, 2018.

[24] J. J. H. Reynolds, M. Torremorell, and M. E. Craft, "Mathematical modeling of influenza a virus dynamics within swine farms and the effects of vaccination," PLoS One, vol. 9, no. 8, Article ID 0106177, 2014.

[25] L. Mitchell and J. V. Ross, "A data-driven model for influenza transmission incorporating media effects," Royal Society Open Science, vol. 3, no. 10, Article ID 160481, 2016. 
[26] A. O. Egonmwan and D. Okuonghae, "Analysis of a mathematical model for tuberculosis with diagnosis," Journal of Applied Mathematics and Computing, vol. 59, no. 1-2, pp. 129-162, 2019.

[27] L. N. Nkamba, T. T. Manga, F. Agouanet, and M. L. Mann Manyombe, "Mathematical model to assess vaccination and effective contact rate impact in the spread of tuberculosis," Journal of Biological Dynamics, vol. 13, no. 1, pp. 26-42, 2019.

[28] T. Bakary, S. Boureima, and T. Sado, "A mathematical model of malaria transmission in a periodic environment," Journal of Biological Dynamics, vol. 12, no. 1, pp. 400-432, 2018.

[29] E. Irwan, Jaharuddin, and P. Sianturi, "Dynamical system for Ebola outbreak within vaccination treatment," Far East Journal of Mathematical Sciences (FJMS), vol. 102, no. 8, pp. 1711-1726, 2017.

[30] A. Akgül, S. H. A. Khoshnaw, and W. H. Mohammed, "Mathematical model for the Ebola virus disease," Journal of Advanced Physics, vol. 7, no. 2, pp. 190-198, 2018.

[31] S. N. Ainisa, Jaharuddin, and E. H. Nugrahani, "Dynamical system of Zikav disease spread through the isolation with two groups of infected population," Far East Journal of Mathematical Sciences (FJMS), vol. 102, no. 11, pp. 2611-2627, 2017.

[32] S. A. Carvalho, S. O. da Silva, and I. D. C. Charret, "Mathematical modeling of dengue epidemic: control methods and vaccination strategies," Theory in Biosciences, vol. 138, no. 2, pp. 223-239, 2019.

[33] E. O. Omondi, R. W. Mbogo, and L. S. Luboobi, "A mathematical modelling study of HIV infection in two heterosexual age groups in Kenya," Infectious Disease Modelling, vol. 4, pp. 83-98, 2019.

[34] K. C. Chong, C. C. Leung, W. W. Yew et al., "Mathematical modelling of the impact of treating latent tuberculosis infection in the elderly in a city with intermediate tuberculosis burden," Scientific Reports, vol. 9, no. 1, pp. 1-11, 2019.

[35] L. Star and S. M. Moghadas, "The role of mathematical modelling in public health planning and decision making," Purple Paper, National Collaborating Centre for Infectious Diseases, vol. 22, pp. 1-6, 2010.

[36] M. G. Roberts and M. I. Tobias, "Predicting and preventing measles epidemics in New Zealand: application of a mathematical model," Epidemiology and Infection, vol. 124, no. 2, pp. 279-287, 2000.

[37] J. M. Ochoche and R. I. Gweryina, "A mathematical model of measles with vaccination and two phases of infectiousness," IOSR Journal of Mathematics, vol. 10, no. 1, pp. 95-105, 2014.

[38] C. R. MacIntyre, N. J. Gay, H. F. Gidding, B. P. Hull, G. L. Gilbert, and P. B. McIntyre, "A mathematical model to measure the impact of the measles control campaign on the potential for measles transmission in Australia," International Journal of Infectious Diseases, vol. 6, no. 4, pp. 277-282, 2002.

[39] J. Mossong and C. P. Muller, "Modelling measles re-emergence as a result of waning of immunity in vaccinated populations," Vaccine, vol. 21, no. 31, pp. 4597-4603, 2003.

[40] D. Aldila and D. Asrianti, "A deterministic model of measles with imperfect vaccination and quarantine intervention," Journal of Physics: Conference Series, vol. 1218, Article ID 012044, 2019.

[41] J. Wallinga, J. C. M. Heijne, and M. Kretzschmar, “A measles epidemic threshold in a highly vaccinated population," PLoS Medicine, vol. 2, no. 11, Article ID e316, 2005.

[42] S. Verguet, M. Johri, S. K. Morris, C. L. Gauvreau, P. Jha, and M. Jit, "Controlling measles using supplemental immunization activities: a mathematical model to inform optimal policy," Vaccine, vol. 33, no. 10, pp. 1291-1296, 2015.
[43] A. Portnoy, M. Jit, M. Ferrari, M. Hanson, L. Brenzel, and S. Verguet, "Estimates of case-fatality ratios of measles in lowincome and middle-income countries: a systematic review and modelling analysis," The Lancet, vol. 7, no. 4, pp. 472-481, 2019.

[44] S. Edlund, D. Lovett, J. Kaufman, K. Y. Sokat, J. van Wijgerden, and A. J. Poots, "Supporting decision making: modeling and forecasting measles in a London borough," 2018, https://www.biorxiv.org/content/10.1101/ 497800v1.

[45] P. Aaby, C. L. Martins, M.-L. Garly, A. Rodrigues, C. S. Benn, and $\mathrm{H}$. Whittle, "The optimal age of measles immunisation in low-income countries: a secondary analysis of the assumptions underlying the current policy," BMJ Open, vol. 2, no. 4, Article ID e000761, 2012.

[46] S. M. Garba, M. A. Safi, and S. Usaini, "Mathematical model for assessing the impact of vaccination and treatment on measles transmission dynamics," Mathematical Methods in the Applied Sciences, vol. 40, no. 18, pp. 6371-6388, 2017.

[47] E. Simons, M. Mort, A. Dabbagh, P. Strebel, and L. Wolfson, "Strategic planning for measles control: using data to inform optimal vaccination strategies," The Journal of Infectious Diseases, vol. 204, no. 1, pp. S28-S34, 2011.

[48] S. Edward, R. E. Kitengeso, G. T. Kiria, N. Felician, G. G. Mwema, and A. P. Mafarasa, "A mathematical model for control and elimination of the transmission dynamics of measles," Applied and Computational Mathematics, vol. 4, no. 6, pp. 396-408, 2015.

[49] C. Obumneke, I. I. Adamu, and S. T. Ado, "Mathematical model for the dynamics of measles under the combined effect of vaccination and measles therapy," International Journal of Science and Technology, vol. 6, no. 6, pp. 862-874, 2017.

[50] R. Viriyapong and W. Ridbamroong, "Global stability analysis and optimal control of measles model with vaccination and treatment," Journal of Applied Mathematics and Computing, vol. 62, no. 1-2, pp. 207-237, 2019.

[51] P. van den Driessche and J. Watmough, "Reproduction numbers and sub-threshold endemic equilibria for compartmental models of disease transmission," Mathematical Biosciences, vol. 180, no. 2, pp. 29-48, 2002.

[52] C. Castillo-Chaves and B. Song, "Dynamical models of tuberculosis and their applications," Mathematical Biosciences and Engineering, vol. 1, no. 2, pp. 361-404, 2004.

[53] L. S. Pontryagin, V. G. Boltyanskii, R. V. Gamkrelidze, and E. F. Mishchenko, The Mathematical Theory of Optimal Process, vol. 4, Gordon \& Breach, New York, USA, 1986.

[54] D. M. Hamby, "A review of techniques for parameter sensitivity analysis of environmental models," Environmental Monitoring and Assessment, vol. 32, no. 2, pp. 135-154, 1994.

[55] S. Lenhart and J. T. Workman, Optimal Control Applied to Biological Models, CRC Press, Boca Raton, FL, USA, 2007.

[56] S. N. Rayhan, T. Bakhtiar, and Jaharuddin, "Optimal control strategies on a TB-HIV/AIDS confection," International Journal of Ecological Economics and Statistics, vol. 39, no. 3, pp. 28-38, 2018.

[57] K. O. Okosun and O. D. Makinde, "A co-infection model of malaria and cholera diseases with optimal control," Mathematical Biosciences, vol. 258, pp. 19-32, 2014.

[58] B. P. Quiambao, S. R. Gatchalian, P. Halonen et al., "Coinfection is common in measles-associated pneumonia," The Pediatric Infectious Disease Journal, vol. 17, no. 2, pp. 89-93, 1998. 
[59] G. D. Murray and A. D. Cliff, "A stochastic model for measles epidemics in a multi-region setting," Transactions of the Institute of British Geographers, vol. 2, no. 2, pp. 158-174, 1977.

[60] D. Athina, T. Bakhtiar, and Jaharuddin, "Optimal control of malaria transmission in two regions using treatment and spraying," International Journal of Ecological Economics and Statistics, vol. 39, no. 3, pp. 98-108, 2018. 